\title{
Natural Antioxidant Anthocyanins-A Hidden Therapeutic Candidate in Metabolic Disorders with Major Focus in Neurodegeneration
}

\author{
Rahat Ullah ${ }^{1,+}{ }^{,}$Mehtab Khan ${ }^{1,+}$, Shahid Ali Shah ${ }^{1,2}$, Kamran Saeed ${ }^{1}$ and Myeong Ok Kim ${ }^{1, *}$ \\ 1 Division of Applied Life Science (BK 21), College of Natural Sciences, Gyeongsang National University, \\ Jinju 52828, Korea; rahatullah1414@gnu.ac.kr (R.U.); mehtabneuro@gnu.ac.kr (M.K.); \\ alishahshahid@yahoo.com (S.A.S.); kamran.biochem@gnu.ac.kr (K.S.) \\ 2 Department of Chemistry, Sarhad University of Science Information Technology (SUIT), \\ Peshawar Khyber Pakhtunkhwa 25000, Pakistan \\ * Correspondence: mokim@gnu.ac.kr; Tel.: +82-55-772-1345; Fax: +82-55-772-2656 \\ + These authors contributed equally to this paper.
}

Received: 7 May 2019; Accepted: 24 May 2019; Published: 28 May 2019

check for updates

\begin{abstract}
All over the world, metabolic syndrome constitutes severe health problems. Multiple factors have been reported in the pathogenesis of metabolic syndrome. Metabolic disorders result in reactive oxygen species (ROS) induced oxidative stress, playing a vital role in the development and pathogenesis of major health issues, including neurological disorders Alzheimer's disease (AD) Parkinson's disease (PD). Considerable increasing evidence indicates the substantial contribution of ROS-induced oxidative stress in neurodegenerative diseases. An imbalanced metabolism results in a defective antioxidant defense system, free radicals causing inflammation, cellular apoptosis, and tissue damage. Due to the annual increase in financial and social burdens, in addition to the adverse effects associated with available synthetic agents, treatment diversion from synthetic to natural approaches has occurred. Antioxidants are now being considered as convincing therapeutic agents against various neurodegenerative disorders. Therefore, medicinal herbs and fruits currently receive substantially more attention as commercial sources of antioxidants. In this review, we argue that ROS-targeted therapeutic interventions with naturally occurring antioxidant flavonoid, anthocyanin, and anthocyanin-loaded nanoparticles might be the ultimate treatment against devastating illnesses. Furthermore, we elucidate the hidden potential of the neuroprotective role of anthocyanins and anthocyanin-loaded nanoparticles in AD and PD neuropathies, which lack sufficient attention compared with other polyphenols, despite their strong antioxidant potential. Moreover, we address the need for future research studies of native anthocyanins and nano-based-anthocyanins, which will be helpful in developing anthocyanin treatments as therapeutic mitochondrial antioxidant drug-like regimens to delay or prevent the progression of neurodegenerative diseases, such as AD and PD.
\end{abstract}

Keywords: oxidative stress; metabolic syndrome; Alzheimer's disease; Parkinson's disease; anthocyanins; neuroprotection

\section{Introduction}

\subsection{Metabolic Syndrome and Oxidative Stress}

Metabolic syndrome (MetS) is a collection of several risk factors of metabolic disorders that occur together, raising the risk of developing cardiovascular disease and other health problems including type 2 diabetes and stroke. Metabolic syndrome, or syndrome $X$, has been a hot debate in clinical and research settings and has led to mortality and morbidity worldwide. According to the Third Report 
of the National Cholesterol Education Program Adult Treatment Panel III, MetS is characterized by different combinations of three or more features, such as hyperglycemia, a low level of high-density lipoprotein (HDL) cholesterol (HDL-C), hypertriglyceridemia, abdominal obesity, and hypertension [1]. The diagnostic criteria for metabolic syndrome have been defined by the World Health Organization [2], the National Cholesterol Education Program's (NCEP's) Adult Treatment Panel (ATP) III guidelines [3], and another organization [4]. There is a substantial increase in the epidemic proportion of metabolic syndrome (MetS) due to complex interactions between environmental factors and genetic factors, unhealthy dietary habits and sedentary lifestyles [5]. Multiple factors have been reported in the pathogenesis of metabolic syndrome [6]. One candidate, oxidative stress, results in the attack of cellular macromolecules, such as proteins, lipids and nucleic acids, leading to cellular dysfunction [6]. In addition, nutritional stress (i.e., a high fat and high carbohydrate diet) also stimulates oxidative stress, as evident by protein carbonylation, increased lipid peroxidation products, reduced glutathione (GSH) levels, and a decreased antioxidant system. Thus, ROS act as a pathogenic milieu in the development of several chronic diseases, including carcinogenesis, diabetes, obesity, hypertension, cardiovascular diseases, and neurodegenerations. A synergistic interaction between oxidative stress and metabolic disorders (MetS) has emerged, the understanding of which can be helpful in the identification of molecular targets, novel biomarkers, and effective drug development for the prevention and therapy of these diseases.

\subsection{Reactive Oxygen Species (ROS): Sources and Free Radical-Scavenging Mechanisms}

In the fields of biology and medicine, free radicals are known as ROS or reactive nitrogen species (RNS) [7]. ROS encompass a broad category of highly reactive substances that are ubiquitous and short-lived derivatives of oxygen metabolism produced as intermediate products of normal cellular metabolism. ROS can be broadly classified into free radicals and nonradical groups (powerful oxidants). Free radicals consist of unpaired electrons that have short lifetimes (milliseconds-nanoseconds) due to a high energy state and include superoxide $\mathrm{O} 2(-)$ and hydroxyl radicals $(\mathrm{OH}-)$. In contrast, nonradical ROS are relatively stable powerful oxidants that consist of singlet oxygen $\left({ }^{1} \mathrm{O}_{2}\right)$, hypochlorous acid ( $\mathrm{HOCl}$ ), ozone $\left(\mathrm{O}_{3}\right)$, hydrogen peroxide $\left(\mathrm{H}_{2} \mathrm{O}_{2}\right)$, and chloramines ( $\mathrm{RNHCl}$ ) [8]. Similarly, reactive nitrogen species (RNS) that contain nitrogen atoms consist of radical compounds, including nitric oxide (NO), nitroxyl (NO-) anions, peroxynitrite (NO-3), and nonradical compounds, i.e., peroxynitrous acid $\left(\mathrm{HNO}_{3}\right)$ and nitrous oxide $\left(\mathrm{N}_{2} \mathrm{O}\right)$ [9]. Among $\operatorname{ROS}\left(\mathrm{O}_{2} \cdot-{ }_{-},-\mathrm{OH}, \mathrm{H}_{2} \mathrm{O}_{2}\right)$, superoxide $\left(\mathrm{O}_{2}\right.$.- $)$ is highly reactive and a cytotoxic agent.

Endogenous ROS sources comprise intracellular multicompartments (mitochondria, peroxisomes, endoplasmic reticulum, cytosol, nuclei, and plasma membranes), extracellular spaces and enzymes that catalyze ROS-generating chemical reactions (Figure 1) [8]. However, in most mammalian cells, the mitochondrial electron transport chain is the major site of ROS production [10]. In addition, exogenous or external ROS sources include multiple stimuli that accelerate oxidative stress, including tobacco smoke, air pollutants, ionizing and nonionizing radiation, xenobiotics, foods and drugs. Chemical agents, such as quinones [11], organic solvents, heavy metals (lead, chromium) and pesticides, are common exogenous sources of ROS [12]. ROS are also produced in response to ultraviolet (UV) radiation, alcohol consumption, cigarette smoking, ingestion of nonsteroidal anti-inflammatory drugs (NSAIDs), and many other exogenous agents. Ischemia-reperfusion (I/R) injury, infections, and various inflammatory processes also result in elevated levels of ROS [8]. Numerous exogenous and endogenous ROS sources are elucidated in detail in Figure 1. To cope with oxidative stress, human and animal cells have developed a ubiquitous antioxidant system. If the cellular antioxidant system is unable to overcome the amount of generated ROS, the excess ROS will deteriorate the normal cellular homeostasis leading to the oxidation of biological molecules [13]. The resultant oxidized or nitrated products of ROS will decrease biological activity, which leads to metabolic dysregulation and alterations in cell signaling [14] and thus accelerates the pathogenesis of various disease states $[15,16]$. 


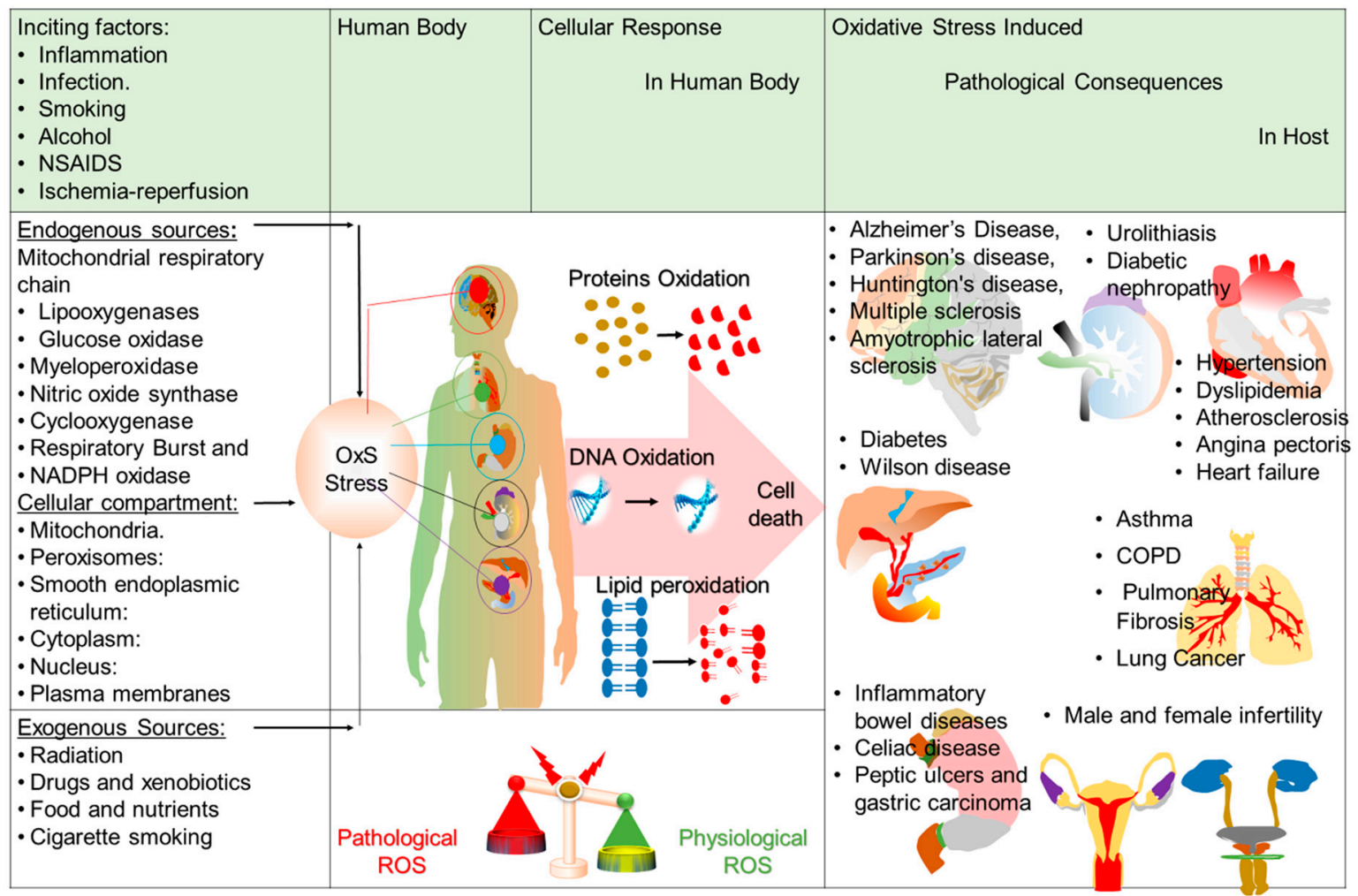

Figure 1. Schematic diagram showing various inciting factors, endogenous and exogenous sources leading to reactive oxygen species (ROS) generation in the human body followed by cellular response leading to pathological consequences in internal organs of the host. NSAIDs: Non-steroidal anti-inflammatory drugs, NADPH: Nicotinamide adenine dinucleotide phosphate, OxS: Oxidative stress, DNA: Deoxyribonucleic acid, COPD: Chronic Obstructive Pulmonary Disease.

An antioxidant may be defined as "a substance" that is present at a lower concentration than an oxidizable substrate and that has the ability to inhibit oxidation of the substrate [17]. For aerobic life, oxidation reactions are important, and abnormal ROS generation is harmful. ROS-induced oxidative stress can be prevented by several mechanisms, such as (i) preventative mechanisms, (ii) physical defense (iii) repair mechanisms, and (iv) antioxidant defenses [18]. Moreover, the body is equipped with antioxidants that act as a shield against the harmful effects of ROS and are collectively termed the antioxidant defense system [8]. This defense system consists of both enzymatic and nonenzymatic mechanisms. The endogenous enzymatic antioxidants include superoxide dismutase (SOD), catalase (CAT), glutathione peroxidase (GPX), glutathione reductase, superoxide reductases and heme oxygenase (HO-1) [19]. In contrast, endogenous nonenzymatic antioxidants include glutathione, thioredoxin, and melatonin. Similarly, exogenous antioxidants include vitamin C, vitamin E, carotenoid, minerals, and polyphenols, including flavonoids [8]. Taken together, collectively these findings indicate, that an imbalance between ROS levels and the antioxidant defense system contribute to the structural and functional loss of biological tissues in the onset and progression of numerous diseases.

\section{Oxidative Stress and Human Disorders}

Accumulating lines of evidence confirm/suggest that oxidative stress plays a vital role in the development and pathogenesis of major health problems leading to cardiovascular diseases [20], joint disorders [21], eye disorders [22], lung and kidney disorders [23,24], atherosclerosis [25], liver and pancreatic diseases [26,27], aging [28], cancer [29], infertility [30], and neurological diseases (Alzheimer's disease, Parkinson's disease and amyotrophic lateral sclerosis) [31]. Herein, we have only focused 
on oxidative stress in $\mathrm{AD}$ and $\mathrm{PD}$, and the previously described disorders are beyond the scope of this review.

\section{Oxidative Stress and Brain}

The brain consumes approximately $20 \%$ of the total amount of the oxygen supply in the body, of which a significant portion is converted to ROS [32]. Evidence from AD or PD brain tissue samples supported elevated oxidative stress signs induced by ROS [33]. Oxidative stress damages all classes of major biomolecules (proteins, lipids, and DNA), and vulnerable neuronal population loss exceeding more than $90 \%$ results in neuronal dysfunction [34].

\subsection{Oxidative Stress and Alzheimer's disease (AD)}

Alzheimer's disease (AD) is a progressive, late-onset dementia and age-dependent neurodegenerative disorder characterized by an intracellular accumulation of tangles (insoluble hyperphosphorylated tau proteins) and extracellular plaques $A \beta$ peptide deposition, i.e., (A $\beta 1-40$ and $A \beta 1-42$ ) [35]. The pathogenesis of $\mathrm{AD}$ has been considered a multifactorial origin [36], and its exact mechanism remains a question of debate. Various factors have been implicated in the etiology of the disease; oxidative stress is among one of the widely accepted factors [37]. Numerous studies have reported that oxidative stress is an early response in both the human AD brain [38] and animal models of AD, where it accelerates $A \beta$ accumulation [39], suggesting a central role of oxidative stress in the early episode of AD pathogenesis [40]. Similarly, reactive nitrogen species (RNS) have also been found to contribute to AD pathology by aberrantly activating N-methyl-D-aspartate receptors (NMDARs) by glutamate (excitotoxicity) [7].

\subsubsection{Sources of Oxidative Stress in AD}

In $\mathrm{AD}$, in addition to metabolically derived ROS, there are a number of additional contributory sources that play an important role in disease progression. The major sources of oxidative stress include mitochondrial, $A \beta$, glial cell, advanced glycation end product (AGE) altered cellular signaling, and metal abnormalities (Figure 2).

\section{Anthocyanin in AD}

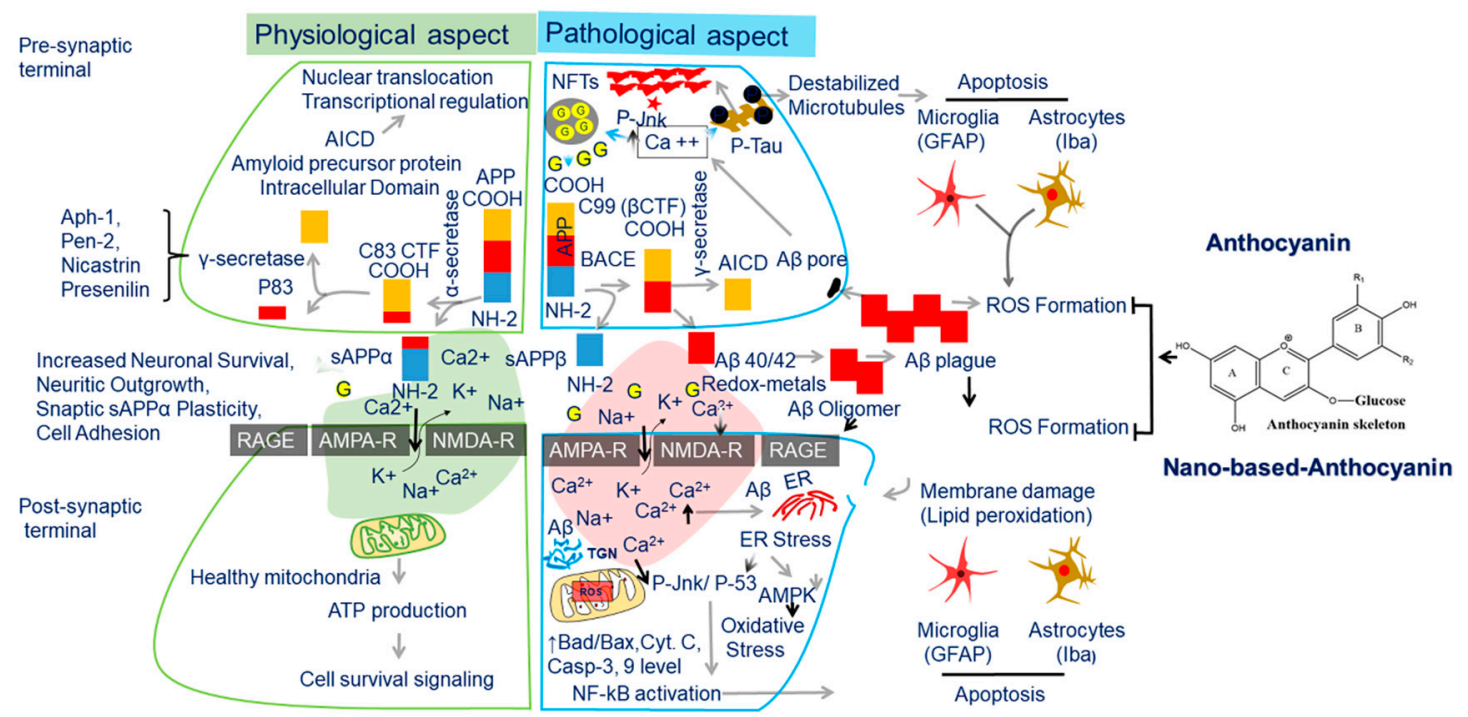

Figure 2. The schematic representation of physiological and pathological aspects (major sources of oxidative stress) in Alzheimer's disease (AD) and their corresponding antioxidant defense approach by anthocyanin in cholinergic neurons. Mitochondrial abnormalities, advanced glycation end products (RAGE), redox-active metals (iron, copper and zinc), amyloid beta $(\mathrm{A} \beta)$ deposition, and alterations in 
cell signaling pathways are major sources of Oxidative stress (OxS) in AD. In an amyloidogenic pathway, the generated neurotoxic $A \beta 40 / 42$ peptides from integral membrane protein Amyloid precursor Protein (APP) after sequential cleavage by $\beta$ and $\gamma$-secretase is aggregated and formed oligomers before plaque (extracellularly) and neurofibrillary tangles (intracellularly) formation. This results in the disruption of calcium homeostasis, blocked ion channels, mitochondrial dysfunction impairment of energy metabolism, and glial cell activation that ultimately leads to oxidative stress resulting in neuronal cell death. In contrast, in a non-amyloidogenic pathway, APP is initially cleaved by $\alpha$-secretase followed by further cleavage by $\gamma$-secretase excluding the possibility of $A \beta$ generation and thus ROS production. Anthocyanin inhibited generated oxidative stress (OxS) in AD providing a neuro-protective effect. Abbreviations. BACE1, beta-site APP cleaving enzyme 1; $\beta C T F$, Beta site intracellular carboxyl terminal fragment; AICD, amyloid intracellular domain; APP, Amyloid precursor protein, GFAP, Glial fibrillary acidic protein, Iba-1, ionized calcium adaptor binding molecule 1, NF- $\mathrm{kB}$, Nuclear factor- $\mathrm{kB}$, p- JNK, phospo c-Jun N-terminal Kinase.

Mitochondrial Abnormalities

The mitochondria and its role in the decision of cell fate to live or die is of substantial importance [41]. Evidence suggests that mitochondria have a dominant role in ROS production [42]. In AD, mitochondrial abnormalities/damage due to the deficiency of several key enzymes, including $\alpha$-ketoglutarate dehydrogenase complex (KGDHC) and pyruvate dehydrogenase complex (PDHC), lead to ROS production $[43,44]$. Oxidative stress damages mitochondria and thus causes mitochondrial dysfunction, which ultimately induces apoptotic cell death followed by neurodegeneration [45]. In AD, the ranking of factors that contribute to mitochondrial dysfunction includes (i) low vascular blood flow to the brain due to chronic hypoxia/hypoperfusion [46], (ii) enhanced sporadic mutations in mtDNA [47], and (iii) a direct $A \beta$ accumulation that occurs in mitochondria where it is processed in combination with amyloid- $\beta$ protein precursor $(\mathrm{A} \beta \mathrm{PP})[48,49]$. Reddy et al. also reported $\mathrm{A} \beta$ induced mitochondrial dysfunction in AD [50]. Furthermore, hyperhomocysteinemia, homocysteine [51,52] and apolipoprotein E4 (ApoE4) [53] are strong factors involved in mitochondrial dysfunction leading to AD development.

\section{Glial Cell Activation}

In the AD brain, senile plaques and neurofibrillary tangles (NFT) or injured neurons provoke inflammation in a similar fashion as inflammation that occurs in damaged peripheral cells or tissues. A $\beta$ deposition activates glial cells [54], which express a wide range of inflammatory mediators, including cyclooxygenase, chemokines, and cytokines [55]. Studies have demonstrated a direct link between activated NADPH oxidase of microglia and $\mathrm{A} \beta$ peptide, resulting in the burst release of superoxide radicals and the enhanced production of hydrogen peroxide [56,57]. Nitric oxide (NO) produced by activated astrocytes and microglia reacts with superoxide to form peroxynitrite, thus leaving nitrotyrosine, i.e., an identifiable marker. The excess production of nitric oxide (NO) in AD can be measured from the increased amount of nitrotyrosine-modified proteins [58]. In the AD brain, amyloid plaques are surrounded by activated astrocytes with a high iNOS level [59,60]. In contrast, activated microglia involve the enzyme myeloperoxidase (MPO) in ROS production [61]. Myeloperoxidase (MPO) has an active role in the formation of nitrotyrosine-modified protein formation [62] and advanced glycation end product modifications [63], both of which are evident in AD.

\section{Advanced Glycation End Products}

In the Alzheimer's disease (AD) brain, the accumulation of advanced glycation end products (AGEs) has been reported and is considered a feature of aging [64]. AGEs undergo redox cycling and play a vital role in ROS production in the presence of transition metals, thus promoting neuronal dysfunction in Alzheimer's disease [65,66]. Similarly, other convincing evidence also supports the prominent role of AGEs and amyloid- $\beta$ in ROS production by acting via RAGE, i.e., receptor for advance glycation end product [67]. 
Redox-Active Metals: Iron, Copper, and Zinc

The brain has a relatively high concentration of biometals (iron, copper, and zinc). Studies have confirmed that abnormal homeostatic mechanisms of copper, iron, and zinc accelerate redox-metal binding to $A \beta$ and tau proteins in $A D$ [68] via the Fenton reaction. For example, iron-induced-oxidative stress pledges various apoptotic signaling pathways, leading to synaptic dysfunction and neuronal cell death [69]. Similarly, copper binds with $A \beta$ to form $A \beta$ : Cu complexes, as well as its inappropriate binding with tau protein, trigger oxidative stress [70-72]. Moreover, zinc is hypothesized to be involved in APP processing [73], leading to greater A $\beta$ deposition [74]. Similarly, zinc binding to tau protein promotes its aggregation and thus accelerates tau-related neuropathology in AD [75].

\section{Amyloid beta $(\mathrm{A} \beta)$ Deposition}

Many researchers highlight the central role of $A \beta$-induced-oxidative stress in the pathogenesis of the Alzheimer's disease (AD) brain [76,77]. In the amyloidogenic pathway, APP is first proteolytically cleaved by $\beta$-secretase (BACE1, beta-site APP cleaving enzyme 1), which results in the extracellular soluble sAPP $\beta$ (ectodomain) and an intracellular carboxyl terminal fragment ( $\beta C T F)$. The latter is then further cleaved by $\gamma$-secretase inside the cell membrane, releasing A $\beta$ peptides (neurotoxic) and AICD, i.e., amyloid intracellular domain. However, in the non-amyloidogenic pathway, APP is first cleaved by $\alpha$-secretase, resulting in soluble $\operatorname{sAPP} \alpha$ (neuroprotective roles in synaptic plasticity and neuronal survival) and $\alpha \mathrm{CTF}$, i.e., membrane bound. The membrane bound $\alpha \mathrm{CTF}$ is further cleaved by $\gamma$-secretase, releasing P83 peptide (shorter fragment) and AICD. Therefore, it excludes the possibility of $A \beta$ generation [78]. Studies have reported that $A \beta 1-42$ is more toxic than $A \beta 1-40$, which play a vital role in hydrogen peroxide or ROS production [79]. Oxidative damage stimulates $\beta$-secretase and thus elevates $A \beta 1-42$ levels $[80,81]$. Similarly, $A \beta$ deposition has also been found in mitochondrial dysfunction in $\mathrm{AD}$ [82]. $\mathrm{A} \beta$ is also involved in cell membrane perforation by enhancing the calcium influx in cultured hippocampal neurons [83]. The increase in calcium influx triggers presynaptic glutamate neurotransmitter release, which activates postsynaptic N-Methyl-D-aspartic acid (NMDA) receptors [78] (Figure 2).

Upon the binding of excessive glutamate to N-methyl-D-aspartate (NMDA) receptors, i.e., ionotropic receptors, these receptors become permeable to calcium, potassium, and sodium ions. The abnormal intracellular $\mathrm{Ca}+2$ levels induce mitochondrial damage and ROS production by increasing the mitochondrial Ca+2 burden [84]. Most importantly, Shahid et al. [85] reported that exogenously administered glutamate enhanced ROS production by activating AMPK and increasing the intracellular calcium level and expression levels of apoptotic and proinflammatory markers, such as caspase-3, COX2 and p-NF-kB, in both in vivo and in vitro models of glutamate-induced oxidative stress; this production is abrogated by anthocyanins.

A relationship between $A \beta$ oligomers and oxidative stress via NMDA receptor overactivation has been reported [86]. A $\beta$ oligomers are responsible for an abnormal calcium influx, the activation of calpain, and degradation of dynamin 1 via NMDA receptors [87]. Taken together, this evidence suggests that the dysregulation of NMDA-R function induced by $\mathrm{A} \beta$ oligomers caused mitochondrial dysfunction followed by excessive ROS formation [86]. Moreover, A $\beta$ has been reported as directly producing hydrogen peroxide via metal ion reduction [70]. Similarly, an increased level of 8-hydroxy-2-deoxyguanosine (8-OHdG), a marker of DNA oxidation, has been well documented in $\mathrm{AD}$ [88]. Overall, these effects of $A \beta$ alter cellular function, resulting in neurotoxicity.

\section{Alterations in Cell Signaling Pathways}

It is quite clear that oxidative stress induces alterations in the expression and activities of enzymes interacting within multiple signaling pathways. Among these pathways, stress-activated protein kinase (SAPK) pathways are the central mediators that play a vital role in amplifying stress signals into the nucleus. Therefore, in AD, oxidative stress signaling events cause JNK/SAPK activation and 
precede amyloid deposition [89]. Similarly, studies from multiple research groups have reported that $A \beta$ induces a two- to three-fold increase in the activation of JNK/SAPK in multiple neuronal cell lines [90-92]. This activation of the JNK/SAPK pathway causes lipid peroxidation [93] and alterations of antioxidant enzymes, i.e., HO-1 and SOD1, in AD [94,95], and it upregulates BACE-1 expression, followed by elevated $A \beta$ peptide levels, synaptic dysfunction, and neuronal cell death in AD $[96,97]$. Similarly, both in vitro and in vivo studies have reported stress-activated protein kinase (SAPK/JNK-P) in tauopathies [98]. Similar to reports on JNK/c-Jun, other studies highlight that oxidative stress increases the expression of Jnk/p38 levels and its strong association with senile plaque neurites and NFT in the pathogenesis of AD [89,99], as shown in (Figure 2).

\subsection{Oxidative Stress and Parkinson's Disease}

Parkinson's disease (PD) is a chronic, progressive and the second most common neurological disease characterized by a loss of dopaminergic neurons (DA) in the substantia pars compacta (SNpc) and intracellular inclusion bodies ( $\alpha$-synuclein) [100]. The exact etiology of PD remains unclear, and understanding the precise mechanisms is the subject of intensive research.

\subsubsection{Sources of Oxidative Stress in PD}

There are a number of additional contributory sources that play an important role in the disease progression. However, the major sources of oxidative stress include dopamine metabolism, mitochondrial dysfunction, and neuroinflammation (Figure 3).

\section{Dopamine Metabolism}

In a normal physiological state, dopamine is released from presynaptic neurons into the synaptic cleft that activates dopaminergic receptors (D1 and D2) located on postsynaptic dopaminergic neurons. Dopamine (DA) is synthesized from tyrosine by tyrosine hydroxylase (TH) and is stored in synaptic vesicles, where its concentration is maintained in the physiological range. However, in the pathological state, when the cytosolic DA level is increased outside of the synaptic vesicle, e.g., metabolism or by autooxidation, it produces quinones, i.e., o-quinone, 5,6-indolequinone, and aminochrome [101]. DA quinones cause alterations in PD-related proteins, such as $\alpha$-synuclein ( $\alpha$-syn), parkin (E3 ubiquitin protein ligase), DJ-1 (PARK7), superoxide dismutase-2 (SOD2), and UCH-L1 (ubiquitin carboxyl-terminal hydrolase isozyme L1) [101-103], which result in the inactivation of TH enzyme [104] causing brain mitochondrial dysfunction $[105,106]$. Similarly, under iron overload conditions, these quinones convert into neuromelanin involved in ROS production and [107] also exacerbate the neuroinflammatory process by microglial activation in PD [101]. In summary, dopamine itself directly or indirectly via its metabolites (quinones) and neuromelanin enhances mitochondrial ROS production (oxidative stress) or microglial activation (neuroinflammation), which ultimately leads to dopaminergic neuronal death in PD.

\section{Mitochondrial Dysfunction}

In PD, mitochondrial dysfunction is closely linked to increased ROS production [108]. Complex I deficiencies or its inhibition have been considered primary sources of ROS production in PD [109]. For example, rotenone and 1-methyl-4-phenyl-1,2,3,6-tetrahydropyridine (MPTP) are two well-known mitochondrial complex I inhibitors that have been reported in DA neuronal cytotoxicity [110]. The toxicity of rotenone has been documented to cause protein oxidation and Lewy body-like formation [111]. Blesa and Przedborski reported the mechanism by which 1-methyl-4-phenyl-1,2,3,6-tetrahydropyridine (MPTP) crosses the blood brain barrier and is oxidized to 1-methyl-4-phenylpyridinium (MPP+), where it accumulates in mitochondria [110]. This compound inhibits complex I in the mitochondrial electron transporter chain (METC) by disrupting electron flow in the mitochondrial electron transporter chain (METC), resulting in decreased ATP production and increased ROS generation [112]. Accumulating lines of evidence have linked genetic mutations of 
several mitochondrial proteins ( $\alpha$-syn, DJ-1, or PINK or parkin) and the promotion of oxidative stress, mitochondrial dysfunction, and DA cell death $[113,114]$. Similarly, multiple lines of evidence also support $\alpha$-syn accumulation (a key constituent of Lewy pathology) inhibited mitochondrial complex I activity leading to ROS-mediated-DA neuronal death in both familial and sporadic PD $[115,116]$. Overall, complex I deficiencies or its inhibition, protein misfolding, genetic mutations in mitochondrial proteins and $\alpha$-synuclein accumulation directly or indirectly affect mitochondrial function and thus play a vital role in the deleterious events involved in PD.

Neuroinflammation

Barcia et al. [117] reported that neuronal loss is linked with chronic neuroinflammation via microglia-resident macrophages in the central nervous system (CNS) in PD. Evidence supports that environmental toxins (neurotoxins, insecticides, or pesticides), endogenous proteins [118], and Parkinson's disease-associated proteins, such as $\alpha$-syn, parkin, DJ-1, and LRRK2 [119], activate microglia that contribute to the neuroinflammatory response by initiating redox signaling in microglia [120]. DA oxidation leads to neuromelanin (NM) formation. Neuromelanin (NM) is released from extracellular dying neuromelanin (NM)-containing dopaminergic neurons in the $\mathrm{SNpc}$. This process is followed by microglial activation, which results in ROS production and chronic neuroinflammation in PD [121,122]. Taken together, these inciting factors are directly or indirectly involved in microglial activation, which contributes to neuroinflammation in PD.

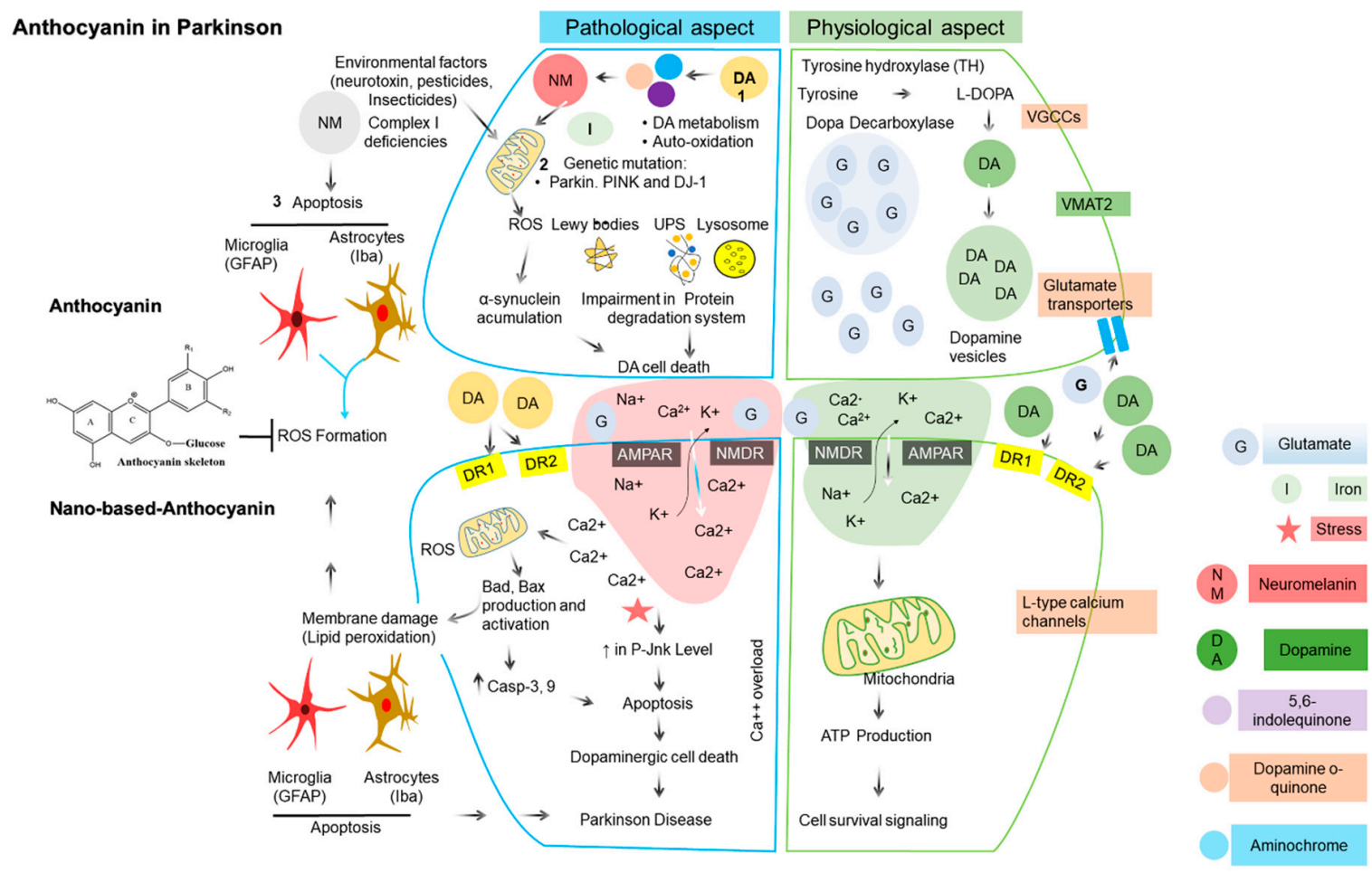

Figure 3. The schematic representation of physiological and pathological aspect (major sources of oxidative stress) in Parkinson's disease (PD) and their corresponding antioxidant defense approach by anthocyanin in dopaminergic neurons. Dopamine metabolism, mitochondrial dysfunction, and neuro-inflammation are major sources of oxidative stress (OxS) in PD. Under the physiological state, release of dopamine (neurotransmitter) and glutamate (after synthesis and storage in their respective synaptic vesicles) are released into the synaptic cleft from presynaptic neuron results in physiological signaling in the postsynaptic neuron by stimulating their respective receptors. In contrast, under the pathophysiological state, oxidative stress $(\mathrm{OxS})$ resulted either from dopamine metabolism, mitochondrial dysfunction and neuro-inflammation from dopaminergic neurons. Anthocyanin combats neuronal oxidative stress (OxS) from the aforementioned sources providing a neuro-protective effect 
in PD. Abbreviations: UPS, ubiquitin proteasome system; $\alpha$-syn, $\alpha$-synuclein; AMPA, $\alpha$-amino-3hydroxy-5-methyl-4-isoxazolepropionate; NMDA, N-methyl-D-aspartate; DR1 and DR2 (dopamine receptor 1 and 2); DA, dopamine, DJ-1, Protein deglycase.

\section{Medications for AD and PD}

\subsection{Synthetic Approaches (Drugs)}

The etiology of both AD and PD is of a multifactorial origin; therefore, the treatment of these diseases is also multitargeted (Tables 1 and 2). Synthetic approaches have been reported that have high costs [123,124], drug-drug interactions, drug-food interactions, and severe side effects [125]. Therefore, to reduce the annual exponential increase in these neurological disorders and overcome their financial and social burdens, neuroscientists are continuously searching for alternative strategies to meet the desired clinical endpoints of affected individuals. That a diet rich in colorful fruits and vegetables reduces the risk of chronic illness is a well-accepted fact, emphasizing the interrelationship between health and diet. The old adage "an apple a day keeps the doctor away" sounds great considering healthcare costs and diet-related chronic illness. Notably, to maintain an active and enjoyable lifestyle as they age, consumers are seeking foods both to prevent disease and to achieve physical and mental health. Such foods provide consumers beneficial physiological effects beyond the required energy and essential nutrients [126]. Due to their health promoting-bioactive compounds, low cost, easy availability, and tolerability as well as offering a broad range of biological activities, including antidementia and cognitive-enhancing effects, fruits and vegetables containing natural antioxidants are currently considered an attractive research subject. 
Table 1. Pharmacological Treatment of AD.

\begin{tabular}{|c|c|c|c|c|c|}
\hline S. No & Group & Classification of AD Drugs & Mechanism of Action & Side Effect & Ref. No. \\
\hline 1 & $\begin{array}{l}\text { Anticholinesterase } \\
\text { Inhibitors (AChEI): (Currently approved } \\
\text { Drugs for AD). }\end{array}$ & $\begin{array}{l}\text { Donepezil(Aricept), } \\
\text { Galantamine (Razadyne), } \\
\text { Rivastigmine (Exelon), } \\
\text { Tacrine(Cognex). }\end{array}$ & Increased Ach level by Inhibiting AchE. & $\begin{array}{l}\text { Nausea, Diarrhea, Vomiting, Loss of appetite, } \\
\text { Abdominal pain, Increased Frequency of bowel } \\
\text { movements, Bradycardia, Hepatotoxicity } \\
\text { (Tacrine/withdrawn from market). }\end{array}$ & [116] \\
\hline 2 & NMDA Receptor Antagonist & Memantine(Namenda) & $\begin{array}{l}\text { Increased } \mathrm{Ca}++ \text { influx (overload) thus } \\
\text { blocking glutamatergic overstimulation. }\end{array}$ & Headache, Constipation, Confusion, and Dizziness. & [127] \\
\hline 3 & $\begin{array}{l}\text { Antihypertensive Drugs } \\
\text {-Diuretics } \\
\text {-Angiotensin -1 Receptor Blockers (ARB): } \\
\text {-Angiotensin-Converting Enzyme } \\
\text { Inhibitors (ACE-I): } \\
\text {-Calcium Channel Blockers (CCB): } \\
\text { Note. Dihydropyridine } \\
\text { are more effective than nondihydropyridine } \\
\text { agents in reducing incidence of AD). } \\
\text {-Beta Blockers }\end{array}$ & $\begin{array}{l}\text { Diuretics: } \\
\text { A.Thiazide Diuretics: } \\
\text { i) Thiazides: } \\
\text {-Hydrochlorohiazie (HCTZ or HCT) } \\
\text { (Apo-Hydro) } \\
\text {-Benzthiazide (Dytide). } \\
\text { ii) Thiazides like: } \\
\text {-Chlorthalidone(Thalitone), } \\
\text {-Metolazone (Zaroxolyn), } \\
\text {-Indapamide (Lozol). } \\
\text { B. Loop Diuretics: } \\
\text {-Furosemide (Lasix), } \\
\text {-Ethacrynic acid (Edecrin). } \\
\text { C. Potassium-sparing Diuretics: } \\
\text { i) Aldosterone Antagonist: } \\
\text {-Spironolactone(Aldactone) } \\
\text {-Eplerenone (Inspra). } \\
\text { ii) Na+ Channel Blocker: } \\
\text {-Amiloride (Midamor) } \\
\text {-Triamterene (Dyrenium). } \\
\text { D.Carbonic Anhydrase Inhibitors: } \\
\text {-Acetazolamide (Diamox), } \\
\text {-Dorzolamide (Trusopt) } \\
\text { Osmotic diuresis: } \\
\text { (Mannitol, Glycerol, Isosorbide and urea). } \\
\text { ARB: } \\
\text {-Candesartan (Atacand), } \\
\text {-Valsartan (Diovan), } \\
\text {-Losartan (Cozaar). } \\
\text { ACE-I:(Centrally acting): } \\
\text {-Captopril(Capoten): } \\
\text {-Ramipril (Altace), } \\
\text {-Lisinopril(Zestril, Prinivil), } \\
\text {-Fosinopril (Monopril). }\end{array}$ & $\begin{array}{l}\text { Diuretics: } \\
\text { Decreased CSF amyloid-beta (A } \beta 1-42) \\
\text { level due to Hypokalemia associated } \\
\text { with diuretics. } \\
\text { ARB:- } \\
\text { Decreased A } \beta \text { production and increased } \\
\text { A } \beta \text { degradation. } \\
\text { ACE-I: } \\
\text { (Controversial) -Increased K+-mediated } \\
\text { acetylcholine release, -Degradation of A } \beta \text {, } \\
\text { Antioxidant potential and vascular } \\
\text { protective effects. }\end{array}$ & $\begin{array}{l}\text { Hyponatremia, dizziness, thirst, muscle cramps } \\
\text { Hypokalemia (except potassium sparing diuretics). } \\
\text { Dizziness, headache, drowsiness, nausea, cough } \\
\text { (low incidence than ACEi), hyperkalemia, muscle } \\
\text { and bone pain. } \\
\text { ACE-I: } \\
\text { Dry cough (due to increase in bradykinin level), } \\
\text { hyperkalemia and fatigue. }\end{array}$ & [128-133] \\
\hline
\end{tabular}


Table 1. Cont.

\begin{tabular}{|c|c|c|c|c|c|}
\hline S. No & Group & Classification of AD Drugs & Mechanism of Action & Side Effect & Ref. No. \\
\hline 3 & $\begin{array}{l}\text { Antihypertensive Drugs } \\
\text {-Diuretics } \\
\text {-Angiotensin -1 Receptor Blockers (ARB): } \\
\text {-Angiotensin-Converting Enzyme } \\
\text { Inhibitors (ACE-I): } \\
\text {-Calcium Channel Blockers (CCB): } \\
\text { Note. Dihydropyridine } \\
\text { are more effective than nondihydropyridine } \\
\text { agents in reducing incidence of AD). } \\
\text {-Beta Blockers }\end{array}$ & $\begin{array}{l}\text { ACE-I:(Non-Centrally acting): } \\
\text {-Benazepril(Lotensin), } \\
\text {-Enalapril(Vasotec), } \\
\text {-Quinapril (Accupril). } \\
\text { CCB: } \\
\text { Dihydropyridine: } \\
\text {-Amlodipine(Norvasc) } \\
\text {-Nifedipine (Adalat). } \\
\text {-Benzothiazepines: } \\
\text {-Diltiazem (Cardizem LA). } \\
\text { Phenylalkylamines: } \\
\text {-Verapamil (Isoptin). } \\
\text { BB: } \\
\text {-Propranolol(Inderal), } \\
\text {-Carvedilol(Coreg), } \\
\text {-Nebivolol(Bystolic). }\end{array}$ & $\begin{array}{l}\text { CCB: } \\
\text {-Decreased intracellular calcium level by } \\
\text { inhibiting calcium channels. } \\
\text {-Decreased A } \beta \text { production. } \\
\text { BB: } \\
\text {-Decreased } \gamma \text { secretase activity } \\
\text { i.e., Aß production. }\end{array}$ & $\begin{array}{l}\text { CCB: } \\
\text { Constipation, flushing, drowsiness, edema and } \\
\text { drowsiness. } \\
\text { Beta Blockers: } \\
\text { Coldness of extremities (hands and feet), dry mouth, } \\
\text { skin and eyes, diarrhea and constipation. } \\
\text { Others: } \\
\text { Congestive Heart Failure, Vivid dreams, } \\
\text { Parasthesias, Depression, Bronchial Bronchospasm, } \\
\text { Night mares and Sexual dysfunction. }\end{array}$ & [128-133] \\
\hline 4 & $\begin{array}{l}\text { NSAIDs. } \\
\text { (Anti-inflammatory drugs) }\end{array}$ & $\begin{array}{l}\text { COX-2 Inhibitor: } \\
\text {-Celecoxib(Celebrex) } \\
\text {-Rofecoxib(Vioxx) } \\
\text {-Valdecoxib (Bextra) } \\
\text { COX-1 Inhibitor: } \\
\text {-lbuprofen (Brufen) } \\
\text {-Aspirin(acetylsalicylicacid) } \\
\text {-Naproxen(Anaprox) } \\
\text {-Flurbiprofen (Anazin) }\end{array}$ & -Decreased neuroinflammation. & Gastrointestinal and Renal toxicity. & [134] \\
\hline 5 & Secretase inhibitors & $\begin{array}{l}\text { BACE inhibitors } \\
\text {-Verubecestat (MK-8931), } \\
\text {-Lanabecestat (AZD3293 or LY3314814), } \\
\text {-Elenbecestat (E2609). } \\
\text { Gamma secretase inhibitor: } \\
\text {-Semagacestat (LY450139) }\end{array}$ & -Decreased $\mathrm{A} \beta$ production. & $\begin{array}{l}\text {-Skin disorders, Small-bowel obstruction, Paranoia, } \\
\text { and Increased agitation }\end{array}$ & {$[135,136]$} \\
\hline
\end{tabular}


Table 1. Cont.

\begin{tabular}{|c|c|c|c|c|c|}
\hline S. No & Group & Classification of AD Drugs & Mechanism of Action & Side Effect & Ref. No. \\
\hline 6 & Insulin & $\begin{array}{l}\text { Insulin Rapid Acting: } \\
\text {-Insulin Lispro (Humalog) } \\
\text {-Insulin Aspart (Novolog) } \\
\text {-Insulin Glulisine (Apidra) } \\
\text { Short-Acting: } \\
\text {-Regular Human (Humulin R) } \\
\text { Intermediate-Acting: } \\
\text {-NPH Human (Humulin N) } \\
\text { Long Acting: } \\
\text {-Insulin Determir (Levemir) } \\
\text {-Insulin Glargine (Lantus) }\end{array}$ & $\begin{array}{l}\text {-Glucose homeostasis -increased A } \beta \\
\text { clearance by enhancing IDE and } \\
\alpha \text {-secretase activity. }\end{array}$ & $\begin{array}{l}\text {-Weight gain, Hypoglycemia, Local reactions } \\
\text { (allergic reactions), Mitogenic properties. }\end{array}$ & {$[137,138]$} \\
\hline 7 & Cytokines & -Etanercept (Enbrel R) (TNF- $\alpha$ modulator) & -Potent antagonist of TNF alpha. & -Headache, Stomach pain, Weakness and Cough. & [139] \\
\hline 8 & HuperzineA (Lycopodium alkaloid) & -HuperzineA & $\begin{array}{l}\text {-Potent inhibitor of AChE } \\
\text {-Anti-oxidant } \\
\text {-Anti-apoptotic } \\
\text {-Anti-inflammatory. }\end{array}$ & -Nausea, Vomiting, Sweating, Blurred vision. & [140] \\
\hline 9 & Polyphenols & -Curcumin, Resveratrol & $\begin{array}{l}\text {-Anti-inflammatory } \\
\text {-Anti-oxidant } \\
\text {-A } \beta \text { clearance-Chelating agent. }\end{array}$ & -Diarrhea, rash, Headache and yellow stool & {$[127,141]$} \\
\hline 10 & Herbal supplements & $\begin{array}{l}\text {-Ginkgobiloba } \\
\text {-Panax gingseng } \\
\text {-Withania somnifera }\end{array}$ & $\begin{array}{l}\text { Ginkgobiloba: Inhibition of mitochondria } \\
\text { dependent apoptosis OR A } \beta \text { aggregation } \\
\text { inhibition. } \\
\text { Panax gingseng: Reduction in } A \beta \text { peptide } \\
\text { level. } \\
\text { Withania Somnifera: Inhibition of AChE, } \\
\text { reduction in of } A \beta \text { level, Antioxidant and } \\
\text { anti-inflammatory activity. }\end{array}$ & -Nausea, Vomiting, Restlessness. & [142-144] \\
\hline 11 & Hormones & -Melatonin/Estrogen (Estrace) & $\begin{array}{l}\text { Melatonin: } \\
\text { Anti-oxidant, } \\
\text { Antiifibrillogenic and Attenuates A } \beta \\
\text { toxicity } \\
\text { Estrogen: Increased growth, survival and } \\
\text { cholinergic activity, antioxidant and } \\
\text { enhanced non-amyloidogenic metabolism } \\
\text { of APP. }\end{array}$ & $\begin{array}{l}\text {-Melatonin: -Depression, Headache, Sleepiness and } \\
\text { irritability. } \\
\text {-Estrogen: } \\
\text { Weight gain and Post-menopausal breast cancer. }\end{array}$ & [145-147] \\
\hline
\end{tabular}


Table 1. Cont.

\begin{tabular}{|c|c|c|c|c|c|c|}
\hline S. No & Group & Classification of AD Drugs & Mechanism of Action & & Side Effect & Ref. No. \\
\hline 12 & $\begin{array}{l}\text { MAO-Is: } \\
\text { (MAO-Ai and MAO-Bi) }\end{array}$ & $\begin{array}{l}\text { MAO-Ai and MAO-Bi (non-sele } \\
\text {-Phenelzine (Nardil), } \\
\text {-Tranylcypromine (Parnate), } \\
\text {-Isocarboxazid (Marplan). } \\
\text { MAO-Ai (Selective): } \\
\text {-Clorgyline (irreversible), } \\
\text {-Moclobemide (reversible). } \\
\text { MAO-Bi (Selective): } \\
\text {-Selegiline(Deprenyl) }\end{array}$ & \multicolumn{2}{|c|}{$\begin{array}{l}\text {-Accelerates nonamyloidogenic pathway. } \\
\text {-Inhibited } A \beta \text { and tau pathophysiology. } \\
\text {-Increased different neurotransmitters level } \\
\text { in brain. }\end{array}$} & $\begin{array}{l}\text { Significant Weight gain, Severe Orthostatic } \\
\text { hypotension, Hypertensive Sexual Dysfunction. }\end{array}$ & {$[148,149]$} \\
\hline 13 & Lipid-lowering drugs & $\begin{array}{l}\text { Statins: } \\
\text {-Lovastatin (Mevacor), } \\
\text {-Simvastatin (Zocor), } \\
\text {-Rosuvastatin (Crestor), } \\
\text { Fibrates: } \\
\text {-Clofibrate (Atromid-S), } \\
\text {-Gemfibrozil (Lopid) } \\
\text { Others: } \\
\text {-Ezetimbe, torcetrapib, } \\
\text {-implitapide. niacin } \\
\end{array}$ & \multicolumn{2}{|c|}{$\begin{array}{l}\text { Statins: } \\
\text { Inhibit HMG-CoA reductase ezyme, and } \\
\text { thus Suppress cholesterol biosynthesis. } \\
\text { Fibrates: } \\
\text { Stimulate } \beta \text {-oxidation of fatty acids } \\
\text {-Lowers plasma level of fatty acid and } \\
\text { triacylglycerol. }\end{array}$} & $\begin{array}{l}\text { Hepatotoxicity Carcinogenic Myopathy } \\
\text { Nephrotoxicity, For-example, Cerivastatin } \\
\text { (Removed from worldwide market due to serious } \\
\text { myopathy/Rhabdomyolysis). }\end{array}$ & {$[150,151]$} \\
\hline 14 & AD Immunotherapy & & \multicolumn{3}{|l|}{$\begin{array}{l}\text {-Use of anti- } \mathrm{A} \beta \text { protein antibodies } \\
\text { (vaccine). }\end{array}$} & [127] \\
\hline S. No. & Classification & Drugs & Mechanism of Action & Side & e Effect & Ref. No. \\
\hline 1. & Dopamine agonists & $\begin{array}{l}\text { Ergot derived dopamine agonists: } \\
\text {-Bromocriptine (Parlodel) } \\
\text {-Pergolide (Permax) } \\
\text {-Cabergoline (Dostinex) } \\
\text { Non-Ergot derived dopamine } \\
\text { agonists: } \\
\text {-Pramipexole (Mirapex) } \\
\text {-Ropinirole (Requip) }\end{array}$ & $\begin{array}{l}\text { The antiparkinsonian effects of } \\
\text { Dopamine agonists (DA) is due to } \\
\text { their direct activation of } \\
\text { dopaminergic receptor (D1 and D2). }\end{array}$ & $\begin{array}{l}\text { Erg } \\
\text { Hall } \\
\text { hyp } \\
\text { skin } \\
\text { vasc } \\
\text { effu } \\
\text { Non } \\
\text { Nau } \\
\text { som }\end{array}$ & $\begin{array}{l}\text { ot-derived-dopamine agonists: } \\
\text { lucination, Delusions, orthostatic, } \\
\text { otension, exacerbation of dyskinesias, } \\
\text { inflammation, erythromelalgia, digital } \\
\text { ospasm, paraesthesias and pleural } \\
\text { sion or pulmonary infiltrates. } \\
\text { l-Ergot-derived dopamine agonists: } \\
\text { isea, Hypotension, Confusion and } \\
\text { nolence and Exacerbation of dyskinesias. }\end{array}$ & {$[152,153]$.} \\
\hline
\end{tabular}


Table 2. Cont.

\begin{tabular}{|c|c|c|c|c|c|}
\hline S. No. & Classification & Drugs & Mechanism of Action & Side Effect & Ref. No. \\
\hline 2. & COMT inhibitors & $\begin{array}{l}\text { Entacapone (Comtan }(), \\
\text { Tolcapone (Tasmarß). }\end{array}$ & $\begin{array}{l}\text {-Inhibition of COMT enzyme in } \\
\text { periphery, thus reducing levodopa } \\
\text { degradation into } 3-O \text { methyldopa } \\
\text { and increased levodopa availability } \\
\text { in brain where it is converted } \\
\text { into dopamine. }\end{array}$ & $\begin{array}{l}\left.\text { Tolcapone (Tasmar }{ }^{\circledR}\right) \text { (black box warning), } \\
\text { Fulminant liver failure, vivid dreams, } \\
\text { diarrhea, urine discoloration(orange), } \\
\text { drowsiness, visualhallucinations } \\
\text { and dyskinesia. }\end{array}$ & {$[154,155]$} \\
\hline 3. & $\begin{array}{l}\text { DOPA decarboxylase } \\
\text { inhibitor (DDCi) }\end{array}$ & $\begin{array}{l}\text { Levodopa (L-DOPA), } \\
\text { Carbidopa (Lodosyn), Or } \\
\text { Combination of both. }\end{array}$ & $\begin{array}{l}\text {-Inhibition of DOPA } \\
\text { decarboxylase in periphery, that } \\
\text { caused levodopa degradation into } \\
\text { dopamine (unable to cross BBB), and } \\
\text { thus enable exogenously } \\
\text { administered DOPA/levodopa to } \\
\text { reach in brain in sufficient quantities, } \\
\text { where it is converted to dopamine. }\end{array}$ & $\begin{array}{l}\text { Motor fluctuations, Dyskinesias ("peak-dose } \\
\text { dyskinesias", "biphasic dyskinesias" and } \\
\text { "wearing-off" dyskinesias }\end{array}$ & {$[153,156]$} \\
\hline 4. & MAO-B Inhibitor & $\begin{array}{l}\text { Selegiline(Eldepryl, Emsam, } \\
\text { Zelapar)-Rasagiline (Azilect) } \\
\text {-Safinamide (Xadago), } \\
\text {-Tranylcypromine } \\
\text { (Parnat, non-selective) }\end{array}$ & $\begin{array}{l}\text { These drugs work by inactivating } \\
\text { MAO enzyme that caused } \\
\text { inactivation of dopamine } \\
\text { neurotransmitter. }\end{array}$ & $\begin{array}{l}\text { High blood pressure, nausea, constipation } \\
\text { Headache, difficulty falling asleep, blurred } \\
\text { vision, cheese reaction (tranylcypromine). }\end{array}$ & [157] \\
\hline 5. & $\begin{array}{l}\text { Anticholinergic } \\
\text { drugs }\end{array}$ & $\begin{array}{l}\text { Trihexyphenidyl (Artane) } \\
\text { Benztropine (Cogentin) } \\
\text { Procyclidine (Kemadrin) } \\
\text { Biperiden (Akineton) }\end{array}$ & $\begin{array}{l}\text { Reduction of persistent tremor or } \\
\text { ameliorate dystonia or dyskinesias } \\
\text { associated with DOPA decarboxylase } \\
\text { inhibitor, or reduction in } \\
\text { extrapyramidal side effects induced } \\
\text { by antipsychotic agents. }\end{array}$ & $\begin{array}{l}\text { Anticholinergic side effects: } \\
\text { Dry mouth, Constipation, } \\
\text { Urinary retention, } \\
\text { Bowel obstruction }\end{array}$ & [158] \\
\hline 6. & Antiviral drug & Amantadine (Symmetrel) & $\begin{array}{l}\text {-Antagonists of NMDA receptor as } \\
\text { well as dopamine re-uptake inhibitor, } \\
\text { therefore increase extracellular } \\
\text { dopamine levels. }\end{array}$ & $\begin{array}{l}\text { Depression, anxiety, hallucination, dry } \\
\text { mouth and constipation. }\end{array}$ & [159] \\
\hline
\end{tabular}

Abbreviations. D1 and D2, Dopaminergic receptors; COMT, Catechol-O-methyltransferase; L-DOPA, levodopa or L-3, 4-dihydroxyphenylalanine; MAO, Monoamine oxidases; NMDAR, N-Methyl-D-aspartate receptor; DOPA decarboxylase inhibitor (DDCi). 


\subsection{Natural Approaches (Antioxidants)}

Epidemiological studies have reported antioxidant compounds with a broad range of biological activities. Antioxidants may be exogenous (natural or synthetic) or endogenous compounds. The natural antioxidant system includes enzymatic (catalase, glutathione peroxidase, and superoxide dismutase (SOD)) and direct-acting nonenzymatic (ascorbic acid, polyphenols, lipoic acid, and carotenoids) compounds and indirectly acting antioxidants that form adducts with metals and thus reverse ROS generation [31]. Natural antioxidants, including polyphenolic compounds obtained from dietary sources, such as anthocyanins from berries, catechins, and theaflavins from tea, curcumin from Curcuma longa or turmeric, resveratrol from grapes and peanuts have received substantial attention in the last few decades due to their broad range of biological activities [160-162].

\section{Anthocyanins: A Natural Antioxidant}

Anthocyanins (a Greek word; anthos means flower and kyanos means blue) are an especially interesting and vigorously studied naturally occurring plant compounds that belong to one of the six subgroups of widespread plant constituents known as flavonoids [163]. The unique ability of anthocyanins to form flavylium cations makes them distinguishable from other flavonoids (Figure 4). The major sources of anthocyanins are blueberries, cherries, strawberries, raspberries, purple grapes, black currants, and red wine [164]. Anthocyanins have gained intensive research interest as a preventive and therapeutic plant agent. Historically, anthocyanin-rich food has been used as traditional medicine [126]. Anthocyanins and anthocyanin-rich extracts have diverse health benefits, leading to their widespread use in certain countries [126,163]. Thus, the old adage may be soon changed to "Binge on berries every day to keep the doctor away". Nevertheless, research studies lack sufficient attention paid to anthocyanin phytochemicals compared to other flavonoids. Therefore, the purpose of this review is to highlight the antioxidant role of anthocyanins and their hidden potential in metabolic disorders, with a special focus on neurodegeneration (AD and PD); antioxidant therapeutic entities alone or in combination are discussed with the aim of overcoming the hazardous effects of current therapies for neurodegenerative disorders in the near future.

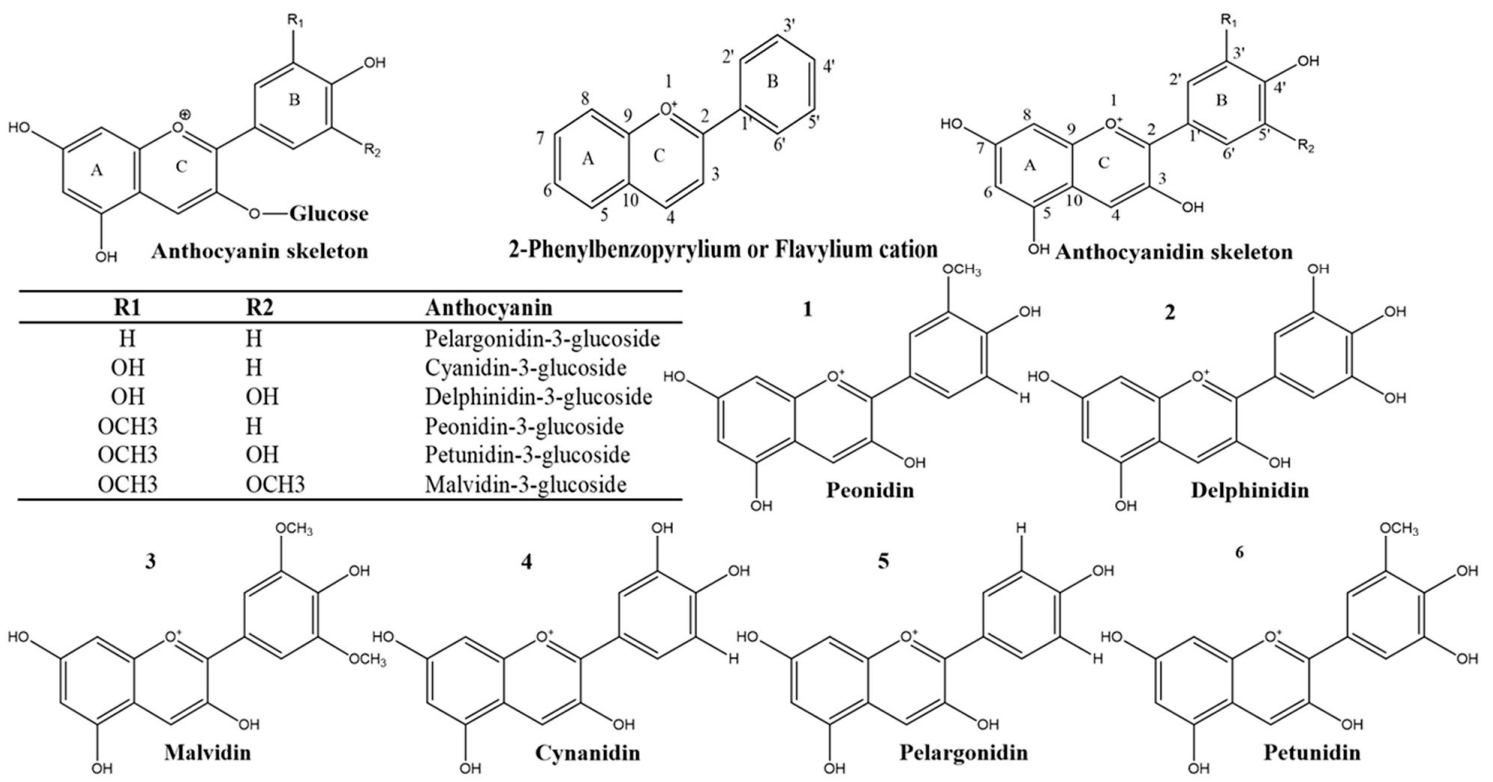

Figure 4. Chemical structures of anthocyanins (glycosylated with glucose), anthocyanidins and 2-phenyl benzopyrylium moiety or flavylium cations: two benzoyl rings (A and B) separated by a heterocyclic (C) ring. (1-6) most common anthocyanidins found in nature; cyanidin (cy), delphinidin $(\mathrm{dp})$ pelargonidin $(\mathrm{pl})$, peonidin (peo), petunidin (pt), and malvidin (mv). 


\subsection{Chemistry, Structure-Activity Relationship and Mode of Action}

In plants, the anthocyanin molecule occurs as a glycoside that contains sugar (glycone moiety, i.e., mostly glucose, or xylose, rhamnose, galactose, or arabinose) and a nonsugar component (aglycone, i.e., anthocyanidin). In anthocyanins, the sugar component is esterified at position 3 , which is missing in anthocyanidin in the same position (Figure 4). Chemically, anthocyanins are glycosylated, polymethoxy or polyhydroxy derivatives of 2-phenyl benzopyrylium moiety and are typically represented as flavylium cations: Two benzoyl rings (A and B) separated by a heterocyclic (C) ring.

To date, over 700 structurally distinct anthocyanins and 27 known anthocyanidin molecules have been identified in nature. The most common anthocyanidins, which account for more than $90 \%$ of the known types, include cyanidins (cy, 50\%), pelargonidins ( $\mathrm{pl}, 12 \%)$, delphinidins ( $\mathrm{dp}, 12 \%)$, petunidins (pt, 7\%), peonidins (peo, 7\%), and malvidins (mv, 12\%) (Figure 4) [126,165]. The glycosylation of the glycone moiety (D-glucose, D-galactose, L-rhamnose, D-xylose, and arabinose) at the C-3 position is most common compared to other carbon positions, i.e., $C-3 / 5 / 7 / 3^{\prime} / 4^{\prime} / 5^{\prime}$, where they are often acylated with aliphatic acids or by cyclic acids [166].

The structure-activity relationship substantially reflects the antioxidant potential of anthocyanins as it is not necessary that all of them possess similar efficacy for scavenging diverse ROS and RNS [167]. Anthocyanin-antioxidant activity is linked with numerous factors, including the number and position of the hydroxyl $(-\mathrm{OH})$ and methoxy $(-\mathrm{OCH} 3)$ groups in the basic anthocyanin skeleton, as well as the type of acylation and glycosylation pattern, e.g., the increased antioxidant effect of anthocyanins has a direct link with the number of the hydroxyl group in human leukemia cells. Similarly, anthocyanins that contain $3^{\prime}, 4^{\prime}$-dihydroxy groups are good metal ion chelators to form stable anthocyanin-metal complexes [166]. In addition to the degree and position of the -OH group in ring $\mathrm{B}$, the position and degree of methoxy $\left(-\mathrm{OCH}_{3}\right)$ also has a greater influence on the anthocyanin-antioxidant potential. For example, according to the results corroborated by Kähkonen and Heinonen (2003), the anthocyanins petunidin-3-glucoside and malvidin-3-glucoside showed a lower efficacy than delphinidin-3-glucoside and cyaniding-3-rutinoside [167]. Furthermore, anthocyanins' antioxidant activity depends on the type of reactive species, i.e., the reactivity against the superoxide anion falls in the order of delphinidin cyanidin pelargonidin, whereas pelargonidin is highly effective against the hydroxyl radical [166].

Anthocyanins may act as extrinsic (direct) or intrinsic (indirect) antioxidants. The direct free radical scavenging effect is due to their potential to donate electron/hydrogen in their structure [166]. Anthocyanins have a high oxygen radical absorption capacity (ORAC) value, which may partially account for the neuroprotective effect $[168,169]$. Similarly, in in vitro models of H2O2 injury and A $\beta$ peptide-induced toxicity, anthocyanins directly scavenge intracellular ROS formation [166,170]. Moreover, the indirect intrinsic antioxidant activity of anthocyanins may be due to (i) the restoration or increase in endogenous antioxidant enzymatic activities (SOD, CAT, and GPx) [171-173] resulting in higher antioxidant glutathione levels [174]; (ii) the activation of these endogenous antioxidants along with phase II detoxification genes by activating the antioxidant response element (ARE) via the redox-sensitive transcription factor NF-E2-related factor-2 (Nrf2) [175,176]; or (iii) the reduction in oxidative adduct formation in DNA and reduction in endogenous ROS formation by inhibiting xanthine oxidase and NADPH oxidase or by modifying arachidonic metabolism and mitochondrial respiration $[165,177]$.

Bioavailability is defined as the quantity or fraction of the ingested dose of a drug or other substance that enters the systemic circulation [178]. It is widely believed that anthocyanins have a low bioavailability. However, recent investigations have shed new light on anthocyanin formulations that have higher bioavailability than those of previous studies. To overcome the bioavailability limitation, anthocyanin-loaded chitosan nanoparticles show a higher bioavailability and stability in the simulated gastrointestinal fluid than free anthocyanins $[179,180]$. Similarly, to increase the bioavailability and stability of anthocyanins due to their hydrophilic unstable nature, nanoparticle-based targeted drug delivery approaches using biodegradable nanoparticle formulations, e.g., PLGA@PEG nanoparticles 
in SH-SY5Y cell lines [181] and PEG-AuNPs in in vivo and in vitro AD models [182], have been used, improving the neurotherapeutic potential of anthocyanins.

\subsection{Pharmacological Activities of Anthocyanins}

Anthocyanins, anthocyanidins, and anthocyanin-rich extracts are of particular interest to nutritionists, due to their diverse health benefits, leading to the widespread use of these compounds in certain countries $[126,163]$. Based on multiple studies, including animal models, cell-line studies, and human clinical trials, it has been concluded that anthocyanins may play a vital role in numerous pathologies, including showing anticancer, antiviral, antidiabetic (via AMPK activation), anti-inflammatory (downregulation of COX-2 and iNOS), cardioprotective (enhancing SOD antioxidant activity), and neuroprotective activities (diminishing glutamate-induced neurotoxicity and increasing glutathione content) (Figure 5) [183,184]. The broad scientific evidence of the beneficial effects of these compounds on human health and their increasing popularity explains their availability in the form of nutraceuticals and food supplements [185].

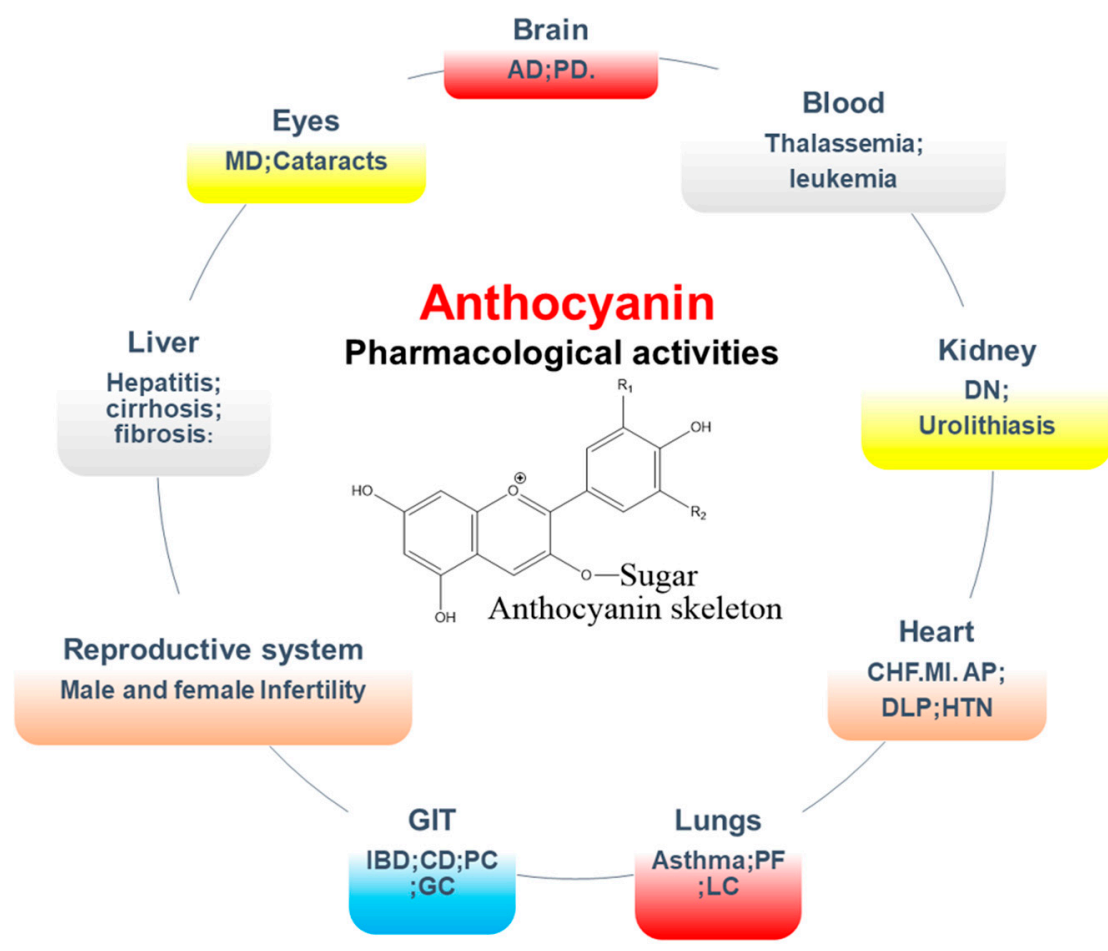

Figure 5. General overview of biological activities of anthocyanins. GIT: Gastrointestinal tract, IBD: inflammatory bowel disease, MD: Macular degeneration, HTN: Hypertension, DLP: Dyslipidemia.

\section{Anthocyanins: A Neuroprotective Agent}

It is known that the brain is the busiest organ and the most sensitive to oxidative stress [31]. Aging, a fundamental cause of brain dysfunction, is accompanied by long-term effects of oxidative stress [186]. Studies have reported the neuroprotective effect of several anthocyanins in AD and PD [187] involving multiple mechanisms, including antioxidant activities [188]. A previous study reported the in vivo antioxidant activity of an orally administered anthocyanin mixture (Vaccinium myrtillis L., $100 \mathrm{mg} / \mathrm{kg}$ bw) against psychological stress-induced cerebral oxidative stress and dopamine abnormalities after psychological stress was induced by cutting off whiskers in distressed mice [189]. Moreover, the strong free radical scavenging effect of purple sweet potato anthocyanins (PSPAs) has been reported against A $\beta$-induced-toxicity in PC12 cells [190]. Similarly, cyanidin-3-O-glucoside isolated from mulberry fruit has been reported with neuroprotection against cerebral ischemia [191]. Notably, in APP1-PS1 transgenic mice and in aged humans, the neuroprotective effect of anthocyanins from blueberry juice 
(12 weeks) and Concord grape juice has been well documented [192,193]. Due to the mounting evidence of the diverse biological activities of anthocyanins, our group [194] reported the neuroprotective effect of anthocyanins against oxidative stress induced by kainic-acid via the AMPK pathway in primary prenatal rat hippocampal neurons and a mouse hippocampal cell line (HT22 cell line) (Figure 6). Similarly, the authors of Reference [195] further explored the neuroprotective effect against ethanol-induced oxidative stress via the GABAB1 receptor intracellular signaling pathway in prenatal hippocampal neurons. The results indicated that anthocyanins significantly inhibited ethanol-induced inhibition of glutamatergic neurotransmission, GABAB1R activation, synaptic dysfunction, and neuronal apoptosis by upregulating the phosphatidylinositol-4,5-bisphosphate 3-kinase PI3K/Akt/ GSK3-beta pathway in the postnatal rat hippocampus brain (P7) and downregulating p-JNK, p-NF-kB, and COX-2 involved in apoptosis in the CA1, CA3 and DG regions of the hippocampus in the developing rat brain (Figure 6). Moreover, the authors of References [196,197] reported similar findings of an anthocyanin-neuroprotective effect against D-galactose and LPS-induced oxidative stress-mediated neurodegeneration in adult rat brains and adult mouse cortex. Furthermore, [198] evaluated the neuroprotective effects of anthocyanins in both in vitro (mouse hippocampal HT22 cells) and in vivo (APP/PS1 mice) mouse models of Alzheimer's disease. More recently, Khan MS [199] extended similar studies and confirmed the antioxidant effect of anthocyanins in in vivo ( 8 week old C57BL/6N mice) and in vitro (HT22 cells and BV2 microglia cells). The results of histological, immunoblot and confocal analyses indicated that the consumption of anthocyanins prevented ROS production, neuroinflammation (by reducing p-NF-kB, IL-1 $\beta$, and TNF- $\alpha$ ), and neuronal apoptosis (as evident from the reduced expression of Bax, cleaved caspase-3, cytochrome $\mathrm{c}$, and cleaved PARP- 1 and increased expression of antiapoptotic Bcl-2 protein). Similarly, anthocyanins increased the levels of survival proteins ( $\mathrm{p}$-Akt, $\mathrm{p}$-GSK3 $\beta$ ) leading to improved hippocampus-dependent memory function (by upregulating memory-related protein levels) and thus preventing neurodegeneration (Figure 6).

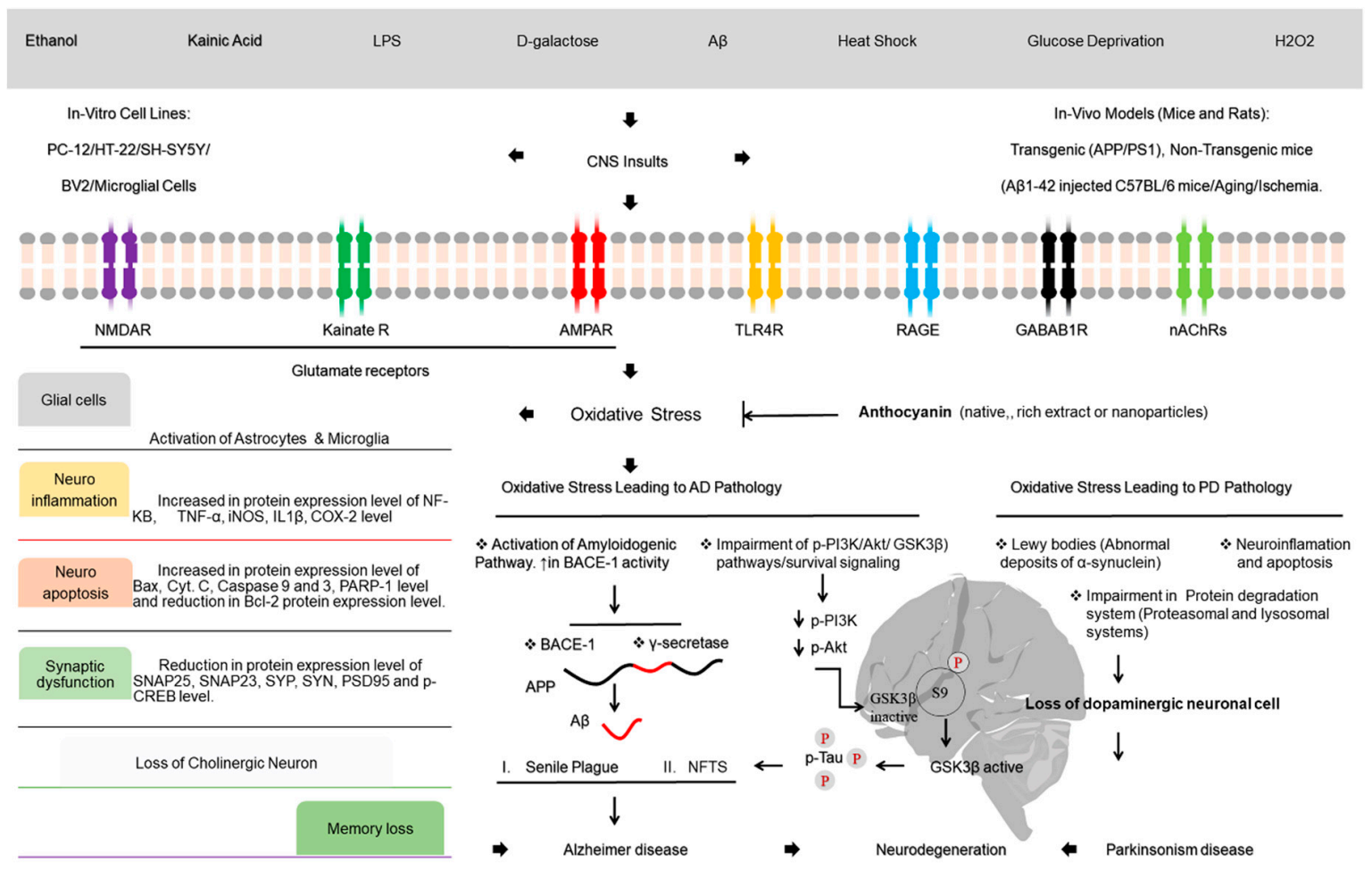

Figure 6. Neuroprotective effect of anthocyanin against oxidative stress by CNS insults (signaling) in AD and PD. p-Tau: phosphorylated Tau Protein.

Globally, Parkinson's disease (PD) is the second most common age associated with disease after AD [200]. Evidence suggests that a polyphenol-rich diet provides neuroprotective properties in 
lowering the risk of PD [201]. Numerous phytochemicals, including green tea [202], curcumin [203], resveratrol and oxyresveratrol [204,205], have been found to be neuroprotective in cellular and animal models of PD [206]. Recent epidemiological studies have reported that the intake of berries, such as strawberries and blueberries, has a neuroprotective effect in PD due to the presence of polyphenols, including anthocyanins and proanthocyanidins [207]. Consistent with these findings, anthocyaninand/or proanthocyanidin-rich extracts also alleviate rotenone induced neurotoxicity by reversing mitochondrial dysfunction in PD [208]. Overall, these observations suggest that anthocyanins act as a neuroprotective agent in lowering the risk of PD and/or slow disease progression.

\section{Nanoparticle Approach of Anthocyanins in Neuroprotection}

Due to the presence of phenolic hydroxyl groups in their chemical structure, anthocyanins are unstable and easily oxidized into quinones, resulting in reduced bioavailability and the loss of biological activity. To overcome these problems and enhance their neuroprotective effect, F.U. Amin et al. [181] for the first time used a nanomedicine therapeutic approach and effectively encapsulated anthocyanins in a biodegradable nanoparticle formulation, i.e., poly(lactide-co-glycolide) (PLGA), using polyethylene glycol (PEG)-2000 as a stabilizing agent; the authors reported their neuroprotective effect via the p38/JNK pathway against A $31-42$-induced oxidative stress in SH-SY5Y cell lines. This nano-based neuroprotective approach of anthocyanins was then further extended in an animal model of Alzheimer's disease by T.Ali et al. [209] using polyethylene glycol-gold nanoparticles (PEG-AuNPs) in an Aßeta1-42 injected mouse model of Alzheimer's disease. Interestingly, the authors found that anthocyanin-loaded PEG-AuNPs (PEG-AuNPs) were more effective than native anthocyanins in reducing the neuropathological disorder of Alzheimer's disease via the PI3K/p-Akt $/ \mathrm{p}-\mathrm{GSK} 3 \beta$ pathway. Similarly, M. J. Kim et al. [188] further evaluated anthocyanin-loaded PEG-gold nanoparticles (PEG-AuNPs) in both in vitro and in vivo models of Alzheimer's disease via the NF-KB /JNK/GSK3 $\beta$ signaling pathway against $\mathrm{A} \beta 1-42$-induced neurodegeneration. Consistent with previous findings, similar findings were obtained using anthocyanin-loaded PEG-AuNPs. Thus, the conjugation of anthocyanins with nanoparticles provides a novel approach that is a helpful, effective, important, and promising strategy in nanomedicine for the prevention of age-associated neurodegenerative diseases. Taken together, this broad impressive evidence/approach opens a future therapeutic window for the encapsulation of anthocyanins (PLGA @ PEG or PEG-AuNPs) and offers a novel, considerable, effective, and promising nanomedicine strategy to improve the efficiency of anthocyanins using nanodrug delivery systems in the field nanomedicine for the prevention AD and PD.

\section{Safety and Toxicological Aspects of Anthocyanins}

Anthocyanins are the most widely consumed flavonoid constituents and their intake is nine-fold higher than that for dietary flavonoids [183]. Accordingly, the use of anthocyanins (E163) in beverages and foods in certain countries, including Japan and the USA, was approved by the Scientific Committee on Food in 1975 and the Joint Food and Agricultural Organization/World Health Organization Expert Committee on Food Additives (JECFA) in 1982 (EFSA, 2013) [210]. Toxicological Reports from the JECFA (1982) concluded that the intake of anthocyanin-rich-extracts is associated with low toxicity based on hematology, clinical parameters, and teratogenic and motility analyses [163,211].

\section{Anthocyanins as Mitochondrial Drug-Like Antioxidants and Their Possible Combinations}

Considerable factors that require particular attention to develop anthocyanins as a mitochondrial drug-like antioxidant include efficacy, safety, stability, favorable pharmacokinetic properties, non-antigenic properties, cell and mitochondria permeability, and having nontoxic metabolites. Notably, anthocyanins as antioxidant molecules must cross the blood-brain barrier (BBB) and preferentially show better oral bioavailability in AD and PD as summarized in Figure 7. 


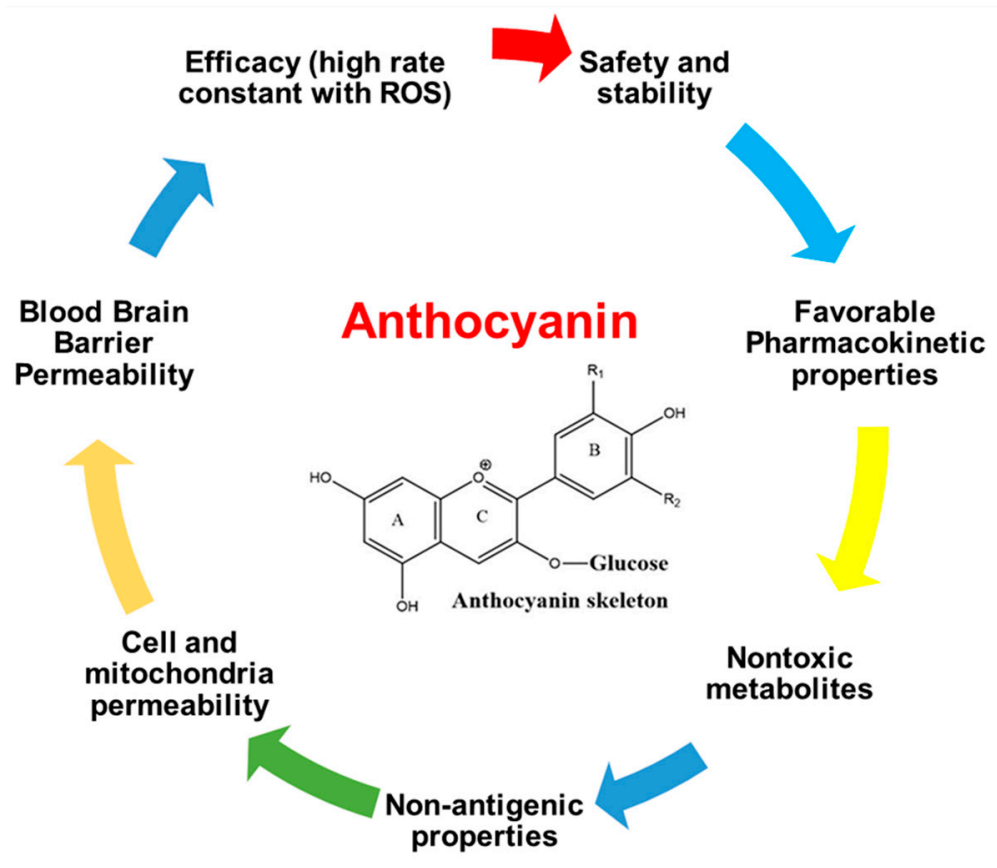

Figure 7. Desirable properties of anthocyanin as a mitochondrial drug-like antioxidant.

Studies have supported antioxidant supplementation alone or in combination with cholinesterase inhibitors to improve the cognitive function in patients with neurological disorders [7]. The antioxidant activity of anthocyanins may be enhanced by other phytochemicals or vitamins that are also abundant in fruits. These compounds may interact with each other synergistically [212]. For example, common dietary flavanols undergo synergistic antioxidant effects with common anthocyanins. M. Rossetto et al. [213] demonstrated a synergistic antioxidant effect of catechin and malvidin 3-glucoside in linoleic acid peroxidation induced by free radicals in micelles. Similarly, the lessons learned from previously synthesized and studied hybrid drugs (acetylcholine esterase inhibitor; tacrine) in combination with antioxidants (melatonin, coumarin, vitamin E) provide hope for a possible therapeutic combination of anthocyanins with a class of anti-AD drugs (Table 1) in the near future [7].

\section{Antioxidants Under Clinical Trials}

To date, more than 635 anthocyanins have been identified in nature [183], among which 26 clinical trials are available (16 completed) that strongly suggest future clinical trials [163]. In human clinical trials, numerous antioxidants are under investigation for their neuroprotective effects. Federally funded Alzheimer's disease centers (an unpublished trial) previously reported that the intake of vitamin E (2000 units/day) and/or selegiline (deprenyl, a MAO-B inhibitor; $10 \mathrm{mg} /$ day) has a slowing effect on AD progression [214]. Similarly, vitamin E, curcumin, and melatonin were found with encouraging results in the reduction of both $A \beta$ tauopathies in in vitro and in vivo AD models [125]. In 2003, Kanowski et al. [215] reported the therapeutic effect of ginkgo biloba special extract EGb 761 ( $240 \mathrm{mg} /$ day) in improving cognitive function in AD patients with dementia. Similar confirmatory findings were subsequently reported using diets (containing vitamin $\mathrm{E}$ alone or in combination with vitamin C or alpha-tocopherol) or antioxidants (Salvia officinalis/Melissa officinalis) in mild to moderate AD patients [125].

Likewise, use of huperzine A in China, and the US initiated its Phase II clinical trial after the joined efforts made by Alzheimer's Disease Cooperative Study and NIH National Institute on Aging. Similarly, Phase II clinical trial of curcumin in AD patients is also instigated by John Douglas French Foundation Institute for the Study of Aging. In early PD patients, Stanley Fahn in 1991, reported an open trial of high-dose antioxidants including vitamin E (DL- $\alpha$ - tocopherol) and ascorbate (vitamin C) for assessment of antioxidants in the brain resulting in slowness to PD progression. Recently, in North 
America, a large multicenter and controlled clinical trial of Deprenyl and Tocopherol Antioxidant Therapy of Parkinsonism (DATATOP) is ongoing for the assessment of an antioxidant (tocopherol; $2000 \mathrm{U} /$ day) used in combination with MAO- B (deprenyl; $10 \mathrm{mg} /$ day) in a total of 800 subjects with early PD. The outcome of this DATATOP study will provide vibrant information regarding the role of antioxidants in the treatment of PD [216].

Taken together, these initial clinical trials on antioxidant and supplements may eventually overlap synthetic treatment and would answer the remaining questions of antioxidants in a clinical trial for $\mathrm{AD}$ and $\mathrm{PD}$ patients in near future.

\section{Conclusion and Future Perspectives}

As neurological disorders are of multi-factorial origin, oxidative stress requires considerable attention as one of the confirmed components, making it a key therapeutic target in combating these neurological disorders. The recent challenges of adverse effects and failed clinical trials, due to a higher toxicity associated with available synthetic compounds for neurological treatments (AD PD), suggest switching to natural compounds as a possible alternative approach. Herein, we argue that targeting oxidative stress with anthocyanins /nano-anthocyanins in diet or supplements as a universal preventive and therapeutic natural-antioxidant drug entity, presents a promising entry point into the aforementioned neuropathies, rather than flooding the body with synthetic compounds that come with side effects.

A nanotechnology-based drug delivery system offering anthocyanin nanoparticles (PLGA@PEG/(PEG-AuNPs) and an encapsulation approach enhanced by pharmacokinetics, pharmacodynamics, and controlled-release profiles in addition to BBB permeability, targets intracellular signaling and, most importantly, ROS scavenging capabilities of anthocyanins in various cellular and animal models in the aforementioned neuropathies. Importantly, it is worth noting that valuable information and lessons learned from failed clinical trials urge the need for the development of desirable 'drug-like' properties and strong rationale that may serve as the future direction for antioxidant drug-development. Going forward, we believe that in-depth analyses and sustained future research efforts are necessary and will open a new therapeutic window for uncovering the dark side (underlying molecular mechanism) of anthocyanins or nano-based-anthocyanins in neuro-protection. Similarly, its synergistic combinations, exploration of composition/particular molecular type/distinct structures, pharmacokinetic, effective functional doses, and metabolite concentrations in the affected brain region are still missing and need to be answered by designing synthetic nano-anthocyanins as therapeutic mitochondrial medicine to delay or prevent the progression of oxidative stress mediated cognitive disorders (AD PD) before entering and meeting their desirable clinical endpoints. The new version of the old adage will soon become "Binge on berries, pop in purple potatoes, revel with radishes, grab the grapes, and wind down with red wine." Enjoy!

Author Contributions: Review design: R.U. Literature revision, data collection, and data analysis: R.U. and M.K. Drafting manuscript: M.K. and K.S. Revising manuscript content: S.A.S. Approving final version of manuscript: M.O.K. and R.U., who take responsibility for the integrity of the data analysis.

Acknowledgments: This review was supported by the Brain Research Program through the National Research Foundation of Korea (NRF) funded by the Ministry of Science, ICT (2016M3C7A1904391).

Conflicts of Interest: The authors declared no competing financial interests.

\section{References}

1. Yubero-Serrano, E.M.; Delgado-Lista, J.; Pena-Orihuela, P.; Perez-Martinez, P.; Fuentes, F.; Marin, C.; Tunez, I.; Tinahones, F.J.; Perez-Jimenez, F.; Roche, H.M.; et al. Oxidative stress is associated with the number of components of metabolic syndrome: LIPGENE study. Exp. Mol. Med. 2013, 45, e28. [CrossRef]

2. Alberti, K.G.; Zimmet, P.Z. Definition, diagnosis and classification of diabetes mellitus and its complications. Part 1: Diagnosis and classification of diabetes mellitus provisional report of a WHO consultation. Diabet. Med. 1998, 15, 539-553. [CrossRef] 
3. Expert Panel on Detection, Evaluation; Treatment of High Blood Cholesterol in Adults. Executive Summary of The Third Report of the National Cholesterol Education Program (NCEP) Expert Panel on Detection, Evaluation, and Treatment of High Blood Cholesterol in Adults (Adult Treatment Panel III). JAMA 2001, 285, 2486-2497. [CrossRef]

4. Alberti, K.G.; Zimmet, P.; Shaw, J.; The IDF Epidemiology Task Force Consensus Group. The metabolic syndrome-A new worldwide definition. Lancet 2005, 366, 1059-1062. [CrossRef]

5. Mokdad, A.H.; Ford, E.S.; Bowman, B.A.; Dietz, W.H.; Vinicor, F.; Bales, V.S.; Marks, J.S. Prevalence of obesity, diabetes, and obesity-related health risk factors, 2001. JAMA 2003, 289, 76-79. [CrossRef]

6. Yadav, U.C.; Rani, V.; Deep, G.; Singh, R.K.; Palle, K. Oxidative Stress in Metabolic Disorders: Pathogenesis, Prevention, and Therapeutics. Oxid. Med. Cell. Longev. 2016, 2016, 9137629. [CrossRef]

7. Poprac, P.; Jomova, K.; Simunkova, M.; Kollar, V.; Rhodes, C.J.; Valko, M. Targeting Free Radicals in Oxidative Stress-Related Human Diseases. Trends Pharmacol. Sci. 2017, 38, 592-607. [CrossRef]

8. Bhattacharyya, A.; Chattopadhyay, R.; Mitra, S.; Crowe, S.E. Oxidative stress: An essential factor in the pathogenesis of gastrointestinal mucosal diseases. Physiol. Rev. 2014, 94, 329-354. [CrossRef]

9. Agarwal, A.; Hamada, A.; Esteves, S.C. Insight into oxidative stress in varicocele-associated male infertility: Part 1. Nat. Rev. Urol. 2012, 9, 678-690. [CrossRef]

10. Poyton, R.O.; Castello, P.R.; Ball, K.A.; Woo, D.K.; Pan, N. Mitochondria and hypoxic signaling: A new view. Ann. N. Y. Acad. Sci. 2009, 1177, 48-56. [CrossRef]

11. Bolton, J.L.; Trush, M.A.; Penning, T.M.; Dryhurst, G.; Monks, T.J. Role of quinones in toxicology. Chem. Res. Toxicol. 2000, 13, 135-160. [CrossRef]

12. Yildirim, A.; Mavi, A.; Oktay, M.; Kara, A.A.; Algur, O.F.; Bilaloglu, V. Comparison of antioxidant and antimicrobial activities of tilia (Tilia argentea Desf ex DC), sage (Salvia triloba L.), and black tea (Camellia sinensis) extracts. J. Agric. Food Chem. 2000, 48, 5030-5034. [CrossRef]

13. Yu, B.P. Cellular defenses against damage from reactive oxygen species. Physiol. Rev. 1994, 74, 139-162. [CrossRef]

14. Chopra, S.; Wallace, H.M. Induction of spermidine/spermine N1-acetyltransferase in human cancer cells in response to increased production of reactive oxygen species. Biochem. Pharmacol. 1998, 55, 1119-1123. [CrossRef]

15. Ames, B.N.; Shigenaga, M.K.; Hagen, T.M. Oxidants, antioxidants, and the degenerative diseases of aging. Proc. Natl. Acad. Sci. USA 1993, 90, 7915-7922. [CrossRef]

16. Wiseman, H.; Halliwell, B. Damage to DNA by reactive oxygen and nitrogen species: Role in inflammatory disease and progression to cancer. Biochem. J. 1996, 313, 17-29. [CrossRef]

17. Halliwell, B.; Gutteridge, J.M. The definition and measurement of antioxidants in biological systems. Free Radic. Biol. Med. 1995, 18, 125-126. [CrossRef]

18. Valko, M.; Leibfritz, D.; Moncol, J.; Cronin, M.T.; Mazur, M.; Telser, J. Free radicals and antioxidants in normal physiological functions and human disease. Int. J. Biochem. Cell Biol. 2007, 39, 44-84. [CrossRef]

19. Lehoux, S. Redox signalling in vascular responses to shear and stretch. Cardiovasc. Res. 2006, 71, $269-279$. [CrossRef]

20. Sugamura, K.; Keaney, J.F., Jr. Reactive oxygen species in cardiovascular disease. Free Radic. Biol. Med. 2011, 51, 978-992. [CrossRef]

21. Afonso, V.; Champy, R.; Mitrovic, D.; Collin, P.; Lomri, A. Reactive oxygen species and superoxide dismutases: Role in joint diseases. Joint Bone Spine 2007, 74, 324-329. [CrossRef]

22. Babizhayev, M.A.; Yegorov, Y.E. Reactive Oxygen Species and the Aging Eye: Specific Role of Metabolically Active Mitochondria in Maintaining Lens Function and in the Initiation of the Oxidation-Induced Maturity Onset Cataract-A Novel Platform of Mitochondria-Targeted Antioxidants With Broad Therapeutic Potential for Redox Regulation and Detoxification of Oxidants in Eye Diseases. Am. J. Ther. 2016, 23, e98-e117. [CrossRef]

23. Rosanna, D.P.; Salvatore, C. Reactive oxygen species, inflammation, and lung diseases. Curr. Pharm. Des. 2012, 18, 3889-3900. [CrossRef]

24. Small, D.M.; Coombes, J.S.; Bennett, N.; Johnson, D.W.; Gobe, G.C. Oxidative stress, anti-oxidant therapies and chronic kidney disease. Nephrology (Carlton) 2012, 17, 311-321. [CrossRef]

25. Harrison, D.; Griendling, K.K.; Landmesser, U.; Hornig, B.; Drexler, H. Role of oxidative stress in atherosclerosis. Am. J. Cardiol. 2003, 91, 7A-11A. [CrossRef] 
26. Li, S.; Tan, H.Y.; Wang, N.; Zhang, Z.J.; Lao, L.; Wong, C.W.; Feng, Y. The Role of Oxidative Stress and Antioxidants in Liver Diseases. Int. J. Mol. Sci. 2015, 16, 26087-26124. [CrossRef]

27. Leung, P.S.; Chan, Y.C. Role of oxidative stress in pancreatic inflammation. Antioxid. Redox Signal. 2009, 11, 135-165. [CrossRef]

28. Muhammad, T.; Ikram, M.; Ullah, R.; Rehman, S.U.; Kim, M.O. Hesperetin, a Citrus Flavonoid, Attenuates LPS-Induced Neuroinflammation, Apoptosis and Memory Impairments by Modulating TLR4/NF- $\mathrm{B}$ Signaling. Nutrients 2019, 11, 648. [CrossRef]

29. Reuter, S.; Gupta, S.C.; Chaturvedi, M.M.; Aggarwal, B.B. Oxidative stress, inflammation, and cancer: How are they linked? Free Radic. Biol. Med. 2010, 49, 1603-1616. [CrossRef]

30. Agarwal, A.; Gupta, S.; Sharma, R.K. Role of oxidative stress in female reproduction. Reprod. Biol. Endocrinol. 2005, 3, 28. [CrossRef]

31. Uttara, B.; Singh, A.V.; Zamboni, P.; Mahajan, R.T. Oxidative stress and neurodegenerative diseases: A review of upstream and downstream antioxidant therapeutic options. Curr. Neuropharmacol. 2009, 7, 65-74. [CrossRef]

32. Chong, Z.Z.; Li, F.; Maiese, K. Oxidative stress in the brain: Novel cellular targets that govern survival during neurodegenerative disease. Prog. Neurobiol. 2005, 75, 207-246. [CrossRef]

33. Kell, D.B. Towards a unifying, systems biology understanding of large-scale cellular death and destruction caused by poorly liganded iron: Parkinson's, Huntington's, Alzheimer's, prions, bactericides, chemical toxicology and others as examples. Arch. Toxicol. 2010, 84, 825-889. [CrossRef]

34. Moreira, P.I.; Smith, M.A.; Zhu, X.; Nunomura, A.; Castellani, R.J.; Perry, G. Oxidative stress and neurodegeneration. Ann. N. Y. Acad. Sci. 2005, 1043, 545-552. [CrossRef]

35. Zhang, Z.; Song, M.; Liu, X.; Su Kang, S.; Duong, D.M.; Seyfried, N.T.; Cao, X.; Cheng, L.; Sun, Y.E.; Ping Yu, S.; et al. Delta-secretase cleaves amyloid precursor protein and regulates the pathogenesis in Alzheimer's disease. Nat. Commun. 2015, 6, 8762. [CrossRef]

36. Benzi, G.; Moretti, A. Are reactive oxygen species involved in Alzheimer's disease? Neurobiol. Aging 1995, 16, 661-674. [CrossRef]

37. Butterfield, D.A.; Reed, T.; Newman, S.F.; Sultana, R. Roles of amyloid beta-peptide-associated oxidative stress and brain protein modifications in the pathogenesis of Alzheimer's disease and mild cognitive impairment. Free Radic. Biol. Med. 2007, 43, 658-677. [CrossRef]

38. Butterfield, D.A.; Swomley, A.M.; Sultana, R. Amyloid beta-peptide (1-42)-induced oxidative stress in Alzheimer disease: Importance in disease pathogenesis and progression. Antioxid. Redox. Signal. 2013, 19, 823-835. [CrossRef]

39. Xie, H.; Hou, S.; Jiang, J.; Sekutowicz, M.; Kelly, J.; Bacskai, B.J. Rapid cell death is preceded by amyloid plaque-mediated oxidative stress. Proc. Natl. Acad. Sci. USA 2013, 110, 7904-7909. [CrossRef]

40. Nunomura, A.; Perry, G.; Aliev, G.; Hirai, K.; Takeda, A.; Balraj, E.K.; Jones, P.K.; Ghanbari, H.; Wataya, T.; Shimohama, S.; et al. Oxidative damage is the earliest event in Alzheimer disease. J. Neuropathol. Exp. Neurol. 2001, 60, 759-767. [CrossRef]

41. Duchen, M.R. Roles of mitochondria in health and disease. Diabetes 2004, 53 (Suppl. 1), S96-S102. [CrossRef]

42. Su, B.; Wang, X.; Nunomura, A.; Moreira, P.I.; Lee, H.G.; Perry, G.; Smith, M.A.; Zhu, X. Oxidative stress signaling in Alzheimer's disease. Curr. Alzheimer Res. 2008, 5, 525-532. [CrossRef]

43. Castellani, R.; Hirai, K.; Aliev, G.; Drew, K.L.; Nunomura, A.; Takeda, A.; Cash, A.D.; Obrenovich, M.E.; Perry, G.; Smith, M.A. Role of mitochondrial dysfunction in Alzheimer's disease. J. Neurosci. Res. 2002, 70, 357-360. [CrossRef]

44. Gibson, G.E.; Sheu, K.F.; Blass, J.P. Abnormalities of mitochondrial enzymes in Alzheimer disease. J. Neural Transm. (Vienna) 1998, 105, 855-870. [CrossRef]

45. Onyango, I.G.; Khan, S.M. Oxidative stress, mitochondrial dysfunction, and stress signaling in Alzheimer's disease. Curr. Alzheimer Res. 2006, 3, 339-349. [CrossRef]

46. De la Torre, J.C. Cerebromicrovascular pathology in Alzheimer's disease compared to normal aging. Gerontology 1997, 43, 26-43. [CrossRef]

47. Coskun, P.E.; Beal, M.F.; Wallace, D.C. Alzheimer's brains harbor somatic mtDNA control-region mutations that suppress mitochondrial transcription and replication. Proc. Natl. Acad. Sci. USA 2004, 101, 10726-10731. [CrossRef] 
48. Lustbader, J.W.; Cirilli, M.; Lin, C.; Xu, H.W.; Takuma, K.; Wang, N.; Caspersen, C.; Chen, X.; Pollak, S.; Chaney, M.; et al. ABAD directly links Abeta to mitochondrial toxicity in Alzheimer's disease. Science 2004, 304, 448-452. [CrossRef]

49. Manczak, M.; Anekonda, T.S.; Henson, E.; Park, B.S.; Quinn, J.; Reddy, P.H. Mitochondria are a direct site of A beta accumulation in Alzheimer's disease neurons: Implications for free radical generation and oxidative damage in disease progression. Hum. Mol. Genet. 2006, 15, 1437-1449. [CrossRef]

50. Reddy, P.H.; Beal, M.F. Amyloid beta, mitochondrial dysfunction and synaptic damage: Implications for cognitive decline in aging and Alzheimer's disease. Trends Mol. Med. 2008, 14, 45-53. [CrossRef]

51. Seshadri, S.; Beiser, A.; Selhub, J.; Jacques, P.F.; Rosenberg, I.H.; D'Agostino, R.B.; Wilson, P.W.; Wolf, P.A. Plasma homocysteine as a risk factor for dementia and Alzheimer's disease. N. Engl. J. Med. 2002, 346, 476-483. [CrossRef] [PubMed]

52. Streck, E.L.; Matte, C.; Vieira, P.S.; Calcagnotto, T.; Wannmacher, C.M.; Wajner, M.; Wyse, A.T. Impairment of energy metabolism in hippocampus of rats subjected to chemically-induced hyperhomocysteinemia. Biochim. Biophys. Acta 2003, 1637, 187-192. [CrossRef]

53. Chang, S.; ran Ma, T.; Miranda, R.D.; Balestra, M.E.; Mahley, R.W.; Huang, Y. Lipid- and receptor-binding regions of apolipoprotein $\mathrm{E} 4$ fragments act in concert to cause mitochondrial dysfunction and neurotoxicity. Proc. Natl. Acad. Sci. USA 2005, 102, 18694-18699. [CrossRef] [PubMed]

54. Eikelenboom, P.; Veerhuis, R. The role of complement and activated microglia in the pathogenesis of Alzheimer's disease. Neurobiol. Aging 1996, 17, 673-680. [CrossRef]

55. Akiyama, H.; Barger, S.; Barnum, S.; Bradt, B.; Bauer, J.; Cole, G.M.; Cooper, N.R.; Eikelenboom, P.; Emmerling, M.; Fiebich, B.L.; et al. Inflammation and Alzheimer's disease. Neurobiol. Aging 2000, 21, 383-421. [CrossRef]

56. Van Muiswinkel, F.L.; Veerhuis, R.; Eikelenboom, P. Amyloid beta protein primes cultured rat microglial cells for an enhanced phorbol 12-myristate 13-acetate-induced respiratory burst activity. J. Neurochem. 1996, 66, 2468-2476. [CrossRef] [PubMed]

57. Klegeris, A.; McGeer, P.L. beta-amyloid protein enhances macrophage production of oxygen free radicals and glutamate. J. Neurosci. Res. 1997, 49, 229-235. [CrossRef]

58. Smith, M.A.; Richey Harris, P.L.; Sayre, L.M.; Beckman, J.S.; Perry, G. Widespread peroxynitrite-mediated damage in Alzheimer's disease. J. Neurosci. 1997, 17, 2653-2657. [CrossRef] [PubMed]

59. Luth, H.J.; Munch, G.; Arendt, T. Aberrant expression of NOS isoforms in Alzheimer's disease is structurally related to nitrotyrosine formation. Brain Res. 2002, 953, 135-143. [CrossRef]

60. Luth, H.J.; Holzer, M.; Gartner, U.; Staufenbiel, M.; Arendt, T. Expression of endothelial and inducible NOS-isoforms is increased in Alzheimer's disease, in APP23 transgenic mice and after experimental brain lesion in rat: Evidence for an induction by amyloid pathology. Brain Res. 2001, 913, 57-67. [CrossRef]

61. Reynolds, W.F.; Rhees, J.; Maciejewski, D.; Paladino, T.; Sieburg, H.; Maki, R.A.; Masliah, E. Myeloperoxidase polymorphism is associated with gender specific risk for Alzheimer's disease. Exp. Neurol. 1999, 155, 31-41. [CrossRef] [PubMed]

62. Podrez, E.A.; Schmitt, D.; Hoff, H.F.; Hazen, S.L. Myeloperoxidase-generated reactive nitrogen species convert LDL into an atherogenic form in vitro. J. Clin. Investig. 1999, 103, 1547-1560. [CrossRef] [PubMed]

63. Anderson, M.M.; Requena, J.R.; Crowley, J.R.; Thorpe, S.R.; Heinecke, J.W. The myeloperoxidase system of human phagocytes generates Nepsilon-(carboxymethyl)lysine on proteins: A mechanism for producing advanced glycation end products at sites of inflammation. J. Clin. Investig. 1999, 104, 103-113. [CrossRef] [PubMed]

64. Munch, G.; Thome, J.; Foley, P.; Schinzel, R.; Riederer, P. Advanced glycation endproducts in ageing and Alzheimer's disease. Brain Res. Brain Res. Rev. 1997, 23, 134-143. [CrossRef]

65. Yan, S.D.; Chen, X.; Schmidt, A.M.; Brett, J.; Godman, G.; Zou, Y.S.; Scott, C.W.; Caputo, C.; Frappier, T.; Smith, M.A.; et al. Glycated tau protein in Alzheimer disease: A mechanism for induction of oxidant stress. Proc. Natl. Acad. Sci. USA 1994, 91, 7787-7791. [CrossRef]

66. Yan, S.D.; Yan, S.F.; Chen, X.; Fu, J.; Chen, M.; Kuppusamy, P.; Smith, M.A.; Perry, G.; Godman, G.C.; Nawroth, P.; et al. Non-enzymatically glycated tau in Alzheimer's disease induces neuronal oxidant stress resulting in cytokine gene expression and release of amyloid beta-peptide. Nat. Med. 1995, 1, 693-699. [CrossRef] 
67. Yan, S.D.; Chen, X.; Fu, J.; Chen, M.; Zhu, H.; Roher, A.; Slattery, T.; Zhao, L.; Nagashima, M.; Morser, J.; et al. RAGE and amyloid-beta peptide neurotoxicity in Alzheimer's disease. Nature 1996, 382, 685-691. [CrossRef]

68. Ayton, S.; Lei, P.; Bush, A.I. Metallostasis in Alzheimer's disease. Free Radic. Biol. Med. 2013, 62, 76-89. [CrossRef]

69. Ayton, S.; Lei, P.; Bush, A.I. Biometals and their therapeutic implications in Alzheimer's disease. Neurotherapeutics 2015, 12, 109-120. [CrossRef]

70. Huang, X.; Atwood, C.S.; Hartshorn, M.A.; Multhaup, G.; Goldstein, L.E.; Scarpa, R.C.; Cuajungco, M.P.; Gray, D.N.; Lim, J.; Moir, R.D.; et al. The A beta peptide of Alzheimer's disease directly produces hydrogen peroxide through metal ion reduction. Biochemistry 1999, 38, 7609-7616. [CrossRef]

71. Opazo, C.; Huang, X.; Cherny, R.A.; Moir, R.D.; Roher, A.E.; White, A.R.; Cappai, R.; Masters, C.L.; Tanzi, R.E.; Inestrosa, N.C.; et al. Metalloenzyme-like activity of Alzheimer's disease beta-amyloid. $\mathrm{Cu}$-dependent catalytic conversion of dopamine, cholesterol, and biological reducing agents to neurotoxic $\mathrm{H}_{2} \mathrm{O}_{2}$. J. Biol. Chem. 2002, 277, 40302-40308. [CrossRef] [PubMed]

72. Su, X.Y.; Wu, W.H.; Huang, Z.P.; Hu, J.; Lei, P.; Yu, C.H.; Zhao, Y.F.; Li, Y.M. Hydrogen peroxide can be generated by tau in the presence of $\mathrm{Cu}(\mathrm{II})$. Biochem. Biophys. Res. Commun. 2007, 358, 661-665. [CrossRef] [PubMed]

73. Maynard, C.J.; Bush, A.I.; Masters, C.L.; Cappai, R.; Li, Q.X. Metals and amyloid-beta in Alzheimer's disease. Int. J. Exp. Pathol. 2005, 86, 147-159. [CrossRef]

74. Bush, A.I.; Pettingell, W.H., Jr.; de Paradis, M.; Tanzi, R.E.; Wasco, W. The amyloid beta-protein precursor and its mammalian homologues. Evidence for a zinc-modulated heparin-binding superfamily. J. Biol. Chem. 1994, 269, 26618-26621. [PubMed]

75. Mo, Z.Y.; Zhu, Y.Z.; Zhu, H.L.; Fan, J.B.; Chen, J.; Liang, Y. Low micromolar zinc accelerates the fibrillization of human tau via bridging of Cys-291 and Cys-322. J. Biol. Chem. 2009, 284, 34648-34657. [CrossRef] [PubMed]

76. Muhammad, T.; Ali, T.; Ikram, M.; Khan, A.; Alam, S.I.; Kim, M.O. Melatonin rescue oxidative stress-mediated neuroinflammation/neurodegeneration and memory impairment in scopolamine-induced amnesia mice model. J. Neuroimmune Pharmacol. 2019, 14, 278-294. [CrossRef] [PubMed]

77. Varadarajan, S.; Yatin, S.; Aksenova, M.; Butterfield, D.A. Review: Alzheimer's amyloid beta-peptide-associated free radical oxidative stress and neurotoxicity. J. Struct. Biol. 2000, 130, 184-208. [CrossRef] [PubMed]

78. Li, Y.; Sun, H.; Chen, Z.; Xu, H.; Bu, G.; Zheng, H. Implications of GABAergic Neurotransmission in Alzheimer's Disease. Front. Aging Neurosci. 2016, 8, 31. [CrossRef] [PubMed]

79. Butterfield, D.A. Amyloid beta-peptide (1-42)-induced oxidative stress and neurotoxicity: Implications for neurodegeneration in Alzheimer's disease brain. A review. Free Radic. Res. 2002, 36, 1307-1313. [CrossRef] [PubMed]

80. Adam-Vizi, V.; Starkov, A.A. Calcium and mitochondrial reactive oxygen species generation: How to read the facts. J. Alzheimers Dis. 2010, 20 (Suppl. 2), S413-S426. [CrossRef]

81. Tamagno, E.; Bardini, P.; Obbili, A.; Vitali, A.; Borghi, R.; Zaccheo, D.; Pronzato, M.A.; Danni, O.; Smith, M.A.; Perry, G.; et al. Oxidative stress increases expression and activity of BACE in NT2 neurons. Neurobiol. Dis. 2002, 10, 279-288. [CrossRef] [PubMed]

82. Swerdlow, R.H.; Khan, S.M. The Alzheimer's disease mitochondrial cascade hypothesis: An update. Exp. Neurol. 2009, 218, 308-315. [CrossRef]

83. Sepulveda, F.J.; Parodi, J.; Peoples, R.W.; Opazo, C.; Aguayo, L.G. Synaptotoxicity of Alzheimer beta amyloid can be explained by its membrane perforating property. PLoS ONE 2010, 5, e11820. [CrossRef] [PubMed]

84. Carriedo, S.G.; Sensi, S.L.; Yin, H.Z.; Weiss, J.H. AMPA exposures induce mitochondrial $\mathrm{Ca}^{2+}$ overload and ROS generation in spinal motor neurons in vitro. J. Neurosci. Off. J. Soc. Neurosci. 2000, 20, $240-250$. [CrossRef]

85. Shah, S.A.; Amin, F.U.; Khan, M.; Abid, M.N.; Rehman, S.U.; Kim, T.H.; Kim, M.W.; Kim, M.O. Anthocyanins abrogate glutamate-induced AMPK activation, oxidative stress, neuroinflammation, and neurodegeneration in postnatal rat brain. J. Neuroinflamm. 2016, 13, 286. [CrossRef] [PubMed]

86. De Felice, F.G.; Velasco, P.T.; Lambert, M.P.; Viola, K.; Fernandez, S.J.; Ferreira, S.T.; Klein, W.L. Abeta oligomers induce neuronal oxidative stress through an N-methyl-D-aspartate receptor-dependent mechanism that is blocked by the Alzheimer drug memantine. J. Biol. Chem. 2007, 282, 11590-11601. [CrossRef] [PubMed] 
87. Kelly, B.L.; Ferreira, A. beta-Amyloid-induced dynamin 1 degradation is mediated by N-methyl-D-aspartate receptors in hippocampal neurons. J. Biol. Chem. 2006, 281, 28079-28089. [CrossRef] [PubMed]

88. Gabbita, S.P.; Lovell, M.A.; Markesbery, W.R. Increased nuclear DNA oxidation in the brain in Alzheimer's disease. J. Neurochem. 1998, 71, 2034-2040. [CrossRef] [PubMed]

89. Zhu, X.; Raina, A.K.; Rottkamp, C.A.; Aliev, G.; Perry, G.; Boux, H.; Smith, M.A. Activation and redistribution of c-jun N-terminal kinase/stress activated protein kinase in degenerating neurons in Alzheimer's disease. J. Neurochem. 2001, 76, 435-441. [CrossRef]

90. Bozyczko-Coyne, D.; O’Kane, T.M.; Wu, Z.L.; Dobrzanski, P.; Murthy, S.; Vaught, J.L.; Scott, R.W. CEP-1347/KT-7515, an inhibitor of SAPK/JNK pathway activation, promotes survival and blocks multiple events associated with Abeta-induced cortical neuron apoptosis. J. Neurochem. 2001, 77, 849-863. [CrossRef] [PubMed]

91. Morishima, Y.; Gotoh, Y.; Zieg, J.; Barrett, T.; Takano, H.; Flavell, R.; Davis, R.J.; Shirasaki, Y.; Greenberg, M.E. Beta-amyloid induces neuronal apoptosis via a mechanism that involves the c-Jun N-terminal kinase pathway and the induction of Fas ligand. J. Neurosci. 2001, 21, 7551-7560. [CrossRef] [PubMed]

92. Troy, C.M.; Rabacchi, S.A.; Xu, Z.; Maroney, A.C.; Connors, T.J.; Shelanski, M.L.; Greene, L.A. beta-Amyloid-induced neuronal apoptosis requires c-Jun N-terminal kinase activation. J. Neurochem. 2001, 77, 157-164. [CrossRef] [PubMed]

93. Pratico, D.; Uryu, K.; Leight, S.; Trojanoswki, J.Q.; Lee, V.M. Increased lipid peroxidation precedes amyloid plaque formation in an animal model of Alzheimer amyloidosis. J. Neurosci. 2001, 21, 4183-4187. [CrossRef] [PubMed]

94. Smith, M.A.; Kutty, R.K.; Richey, P.L.; Yan, S.D.; Stern, D.; Chader, G.J.; Wiggert, B.; Petersen, R.B.; Perry, G. Heme oxygenase- 1 is associated with the neurofibrillary pathology of Alzheimer's disease. Am. J. Pathol. 1994, 145, 42-47. [PubMed]

95. Pappolla, M.A.; Omar, R.A.; Kim, K.S.; Robakis, N.K. Immunohistochemical evidence of oxidative [corrected] stress in Alzheimer's disease. Am. J. Pathol. 1992, 140, 621-628. [PubMed]

96. Tong, Y.; Zhou, W.; Fung, V.; Christensen, M.A.; Qing, H.; Sun, X.; Song, W. Oxidative stress potentiates BACE1 gene expression and Abeta generation. J. Neural Transm. (Vienna) 2005, 112, 455-469. [CrossRef]

97. Tamagno, E.; Parola, M.; Bardini, P.; Piccini, A.; Borghi, R.; Guglielmotto, M.; Santoro, G.; Davit, A.; Danni, O.; Smith, M.A.; et al. Beta-site APP cleaving enzyme up-regulation induced by 4-hydroxynonenal is mediated by stress-activated protein kinases pathways. J. Neurochem. 2005, 92, 628-636. [CrossRef]

98. Rehman, S.U.; Ahmad, A.; Yoon, G.H.; Khan, M.; Abid, M.N.; Kim, M.O. Inhibition of c-Jun N-Terminal Kinase Protects Against Brain Damage and Improves Learning and Memory After Traumatic Brain Injury in Adult Mice. Cereb. Cortex 2018, 28, 2854-2872. [CrossRef]

99. Ferrer, I.; Blanco, R.; Carmona, M.; Puig, B. Phosphorylated mitogen-activated protein kinase (MAPK/ERK-P), protein kinase of $38 \mathrm{kDa}(\mathrm{p} 38-\mathrm{P})$, stress-activated protein kinase (SAPK/JNK-P), and calcium/calmodulin-dependent kinase II (CaM kinase II) are differentially expressed in tau deposits in neurons and glial cells in tauopathies. J. Neural Transm. (Vienna) 2001, 108, 1397-1415. [CrossRef]

100. Blesa, J.; Trigo-Damas, I.; Quiroga-Varela, A.; Jackson-Lewis, V.R. Oxidative stress and Parkinson's disease. Front. Neuroanat. 2015, 9, 91. [CrossRef]

101. Segura-Aguilar, J.; Paris, I.; Munoz, P.; Ferrari, E.; Zecca, L.; Zucca, F.A. Protective and toxic roles of dopamine in Parkinson's disease. J. Neurochem. 2014, 129, 898-915. [CrossRef] [PubMed]

102. Belluzzi, E.; Bisaglia, M.; Lazzarini, E.; Tabares, L.C.; Beltramini, M.; Bubacco, L. Human SOD2 modification by dopamine quinones affects enzymatic activity by promoting its aggregation: Possible implications for Parkinson's disease. PLoS ONE 2012, 7, e38026. [CrossRef] [PubMed]

103. Girotto, S.; Sturlese, M.; Bellanda, M.; Tessari, I.; Cappellini, R.; Bisaglia, M.; Bubacco, L.; Mammi, S. Dopamine-derived quinones affect the structure of the redox sensor DJ-1 through modifications at Cys-106 and Cys-53. J. Biol. Chem. 2012, 287, 18738-18749. [CrossRef] [PubMed]

104. Kuhn, D.M.; Arthur, R.E., Jr.; Thomas, D.M.; Elferink, L.A. Tyrosine hydroxylase is inactivated by catechol-quinones and converted to a redox-cycling quinoprotein: Possible relevance to Parkinson's disease. J. Neurochem. 1999, 73, 1309-1317. [CrossRef] [PubMed]

105. Gluck, M.R.; Zeevalk, G.D. Inhibition of brain mitochondrial respiration by dopamine and its metabolites: Implications for Parkinson's disease and catecholamine-associated diseases. J. Neurochem. 2004, 91, 788-795. [CrossRef] 
106. Jana, S.; Sinha, M.; Chanda, D.; Roy, T.; Banerjee, K.; Munshi, S.; Patro, B.S.; Chakrabarti, S. Mitochondrial dysfunction mediated by quinone oxidation products of dopamine: Implications in dopamine cytotoxicity and pathogenesis of Parkinson's disease. Biochim. Biophys. Acta 2011, 1812, 663-673. [CrossRef] [PubMed]

107. Shamoto-Nagai, M.; Maruyama, W.; Yi, H.; Akao, Y.; Tribl, F.; Gerlach, M.; Osawa, T.; Riederer, P.; Naoi, M. Neuromelanin induces oxidative stress in mitochondria through release of iron: Mechanism behind the inhibition of 26S proteasome. J. Neural Transm. (Vienna) 2006, 113, 633-644. [CrossRef]

108. Schapira, A.H. Mitochondria in the aetiology and pathogenesis of Parkinson's disease. Lancet Neurol. 2008, 7,97-109. [CrossRef]

109. Schapira, A.H.; Cooper, J.M.; Dexter, D.; Clark, J.B.; Jenner, P.; Marsden, C.D. Mitochondrial complex I deficiency in Parkinson's disease. J. Neurochem. 1990, 54, 823-827. [CrossRef]

110. Blesa, J.; Przedborski, S. Parkinson's disease: Animal models and dopaminergic cell vulnerability. Front. Neuroanat. 2014, 8, 155. [CrossRef]

111. Greenamyre, J.T.; Cannon, J.R.; Drolet, R.; Mastroberardino, P.G. Lessons from the rotenone model of Parkinson's disease. Trends Pharmacol. Sci. 2010, 31, 141-142. [CrossRef] [PubMed]

112. Mizuno, Y.; Sone, N.; Saitoh, T. Effects of 1-methyl-4-phenyl-1,2,3,6-tetrahydropyridine and 1-methyl-4-phenylpyridinium ion on activities of the enzymes in the electron transport system in mouse brain. J. Neurochem. 1987, 48, 1787-1793. [CrossRef] [PubMed]

113. Norris, K.L.; Hao, R.; Chen, L.F.; Lai, C.H.; Kapur, M.; Shaughnessy, P.J.; Chou, D.; Yan, J.; Taylor, J.P.; Engelender, S.; et al. Convergence of Parkin, PINK1, and alpha-Synuclein on Stress-induced Mitochondrial Morphological Remodeling. J. Biol. Chem. 2015, 290, 13862-13874. [CrossRef] [PubMed]

114. Van der Merwe, C.; Jalali Sefid Dashti, Z.; Christoffels, A.; Loos, B.; Bardien, S. Evidence for a common biological pathway linking three Parkinson's disease-causing genes: Parkin, PINK1 and DJ-1. Eur. J. Neurosci. 2015, 41, 1113-1125. [CrossRef]

115. Goedert, M.; Spillantini, M.G.; Del Tredici, K.; Braak, H. 100 years of Lewy pathology. Nat. Rev. Neurol. 2013, 9, 13-24. [CrossRef]

116. Jo, M.G.; Ikram, M.; Jo, M.H.; Yoo, L.; Chung, K.C.; Nah, S.Y.; Hwang, H.; Rhim, H.; Kim, M.O. Gintonin Mitigates MPTP-Induced Loss of Nigrostriatal Dopaminergic Neurons and Accumulation of alpha-Synuclein via the Nrf2/HO-1 Pathway. Mol. Neurobiol. 2019, 56, 39-55. [CrossRef] [PubMed]

117. Barcia, C.; Fernandez Barreiro, A.; Poza, M.; Herrero, M.T. Parkinson's disease and inflammatory changes. Neurotox. Res. 2003, 5, 411-418. [CrossRef]

118. Block, M.L.; Zecca, L.; Hong, J.S. Microglia-mediated neurotoxicity: Uncovering the molecular mechanisms. Nat. Rev. Neurosci. 2007, 8, 57-69. [CrossRef]

119. Wilhelmus, M.M.; Nijland, P.G.; Drukarch, B.; de Vries, H.E.; van Horssen, J. Involvement and interplay of Parkin, PINK1, and DJ1 in neurodegenerative and neuroinflammatory disorders. Free Radic. Biol. Med. 2012, 53, 983-992. [CrossRef]

120. Rehman, S.U.; Ali, T.; Alam, S.I.; Ullah, R.; Zeb, A.; Lee, K.W.; Rutten, B.P.F.; Kim, M.O. Ferulic Acid Rescues LPS-Induced Neurotoxicity via Modulation of the TLR4 Receptor in the Mouse Hippocampus. Mol. Neurobiol. 2019, 56, 2774-2790. [CrossRef]

121. Beach, T.G.; Sue, L.I.; Walker, D.G.; Lue, L.F.; Connor, D.J.; Caviness, J.N.; Sabbagh, M.N.; Adler, C.H. Marked microglial reaction in normal aging human substantia nigra: Correlation with extraneuronal neuromelanin pigment deposits. Acta Neuropathol. 2007, 114, 419-424. [CrossRef] [PubMed]

122. Zhang, W.; Phillips, K.; Wielgus, A.R.; Liu, J.; Albertini, A.; Zucca, F.A.; Faust, R.; Qian, S.Y.; Miller, D.S.; Chignell, C.F.; et al. Neuromelanin activates microglia and induces degeneration of dopaminergic neurons: Implications for progression of Parkinson's disease. Neurotox. Res. 2011, 19, 63-72. [CrossRef] [PubMed]

123. Brown, R.C.; Lockwood, A.H.; Sonawane, B.R. Neurodegenerative diseases: An overview of environmental risk factors. Environ. Health Perspect. 2005, 113, 1250-1256. [CrossRef] [PubMed]

124. Meek, P.D.; McKeithan, K.; Schumock, G.T. Economic considerations in Alzheimer's disease. Pharmacotherapy 1998, 18, 68-73, discussion 79-82. [PubMed]

125. Reddy, P.H. Amyloid precursor protein-mediated free radicals and oxidative damage: Implications for the development and progression of Alzheimer's disease. J. Neurochem. 2006, 96, 1-13. [CrossRef] [PubMed]

126. Wallace, T.C.; Giusti, M.M. Anthocyanins in Health and Disease; CRC Press: Boca Raton, FL, USA, 2014.

127. Upadhyay, P.; Panjwani, D.; Yadav, A.K. Neuropathology staging and treatment strategies of Alzheimer's disease: An update. Int. J. Nutr. Pharmacol. Neurol. Dis. 2014, 4, 28. [CrossRef] 
128. DeLoach, T.; Beall, J. Diuretics: A possible keystone in upholding cognitive health. Ment. Health Clin. 2018, 8, 33-40. [CrossRef]

129. Yasar, S.; Xia, J.; Yao, W.; Furberg, C.D.; Xue, Q.L.; Mercado, C.I.; Fitzpatrick, A.L.; Fried, L.P.; Kawas, C.H.; Sink, K.M.; et al. Antihypertensive drugs decrease risk of Alzheimer disease: Ginkgo Evaluation of Memory Study. Neurology 2013, 81, 896-903. [CrossRef]

130. Saavedra, J.M. Evidence to Consider Angiotensin II Receptor Blockers for the Treatment of Early Alzheimer's Disease. Cell Mol. Neurobiol. 2016, 36, 259-279. [CrossRef]

131. Kehoe, P.G. The Coming of Age of the Angiotensin Hypothesis in Alzheimer's Disease: Progress Toward Disease Prevention and Treatment? J. Alzheimers Dis. 2018, 62, 1443-1466. [CrossRef]

132. Goodison, W.V.; Frisardi, V.; Kehoe, P.G. Calcium channel blockers and Alzheimer's disease: Potential relevance in treatment strategies of metabolic syndrome. J. Alzheimers Dis. 2012, 30 (Suppl. 2), S269-S282. [CrossRef]

133. Casey, J. Potassium-sparing diuretics might reduce risk of Alzheimer's disease. Nat. Clin. Pract. Neurol. 2006, 2, 293. [CrossRef]

134. Nivsarkar, M.; Banerjee, A.; Padh, H. Cyclooxygenase inhibitors: A novel direction for Alzheimer's management. Pharmacol. Rep. 2008, 60, 692-698.

135. Fleisher, A.S.; Raman, R.; Siemers, E.R.; Becerra, L.; Clark, C.M.; Dean, R.A.; Farlow, M.R.; Galvin, J.E.; Peskind, E.R.; Quinn, J.F.; et al. Phase 2 safety trial targeting amyloid beta production with a gamma-secretase inhibitor in Alzheimer disease. Arch. Neurol. 2008, 65, 1031-1038. [CrossRef]

136. Vassar, R. BACE1 inhibitor drugs in clinical trials for Alzheimer's disease. Alzheimers Res. Ther. 2014, 6, 89. [CrossRef]

137. Pandini, G.; Pace, V.; Copani, A.; Squatrito, S.; Milardi, D.; Vigneri, R. Insulin has multiple antiamyloidogenic effects on human neuronal cells. Endocrinology 2013, 154, 375-387. [CrossRef]

138. Donner, T. Insulin-Pharmacology, Therapeutic Regimens and Principles of Intensive Insulin Therapy. In Endotext; De Groot, L.J., Chrousos, G., Dungan, K., Feingold, K.R., Grossman, A., Hershman, J.M., Koch, C., Korbonits, M., McLachlan, R., New, M., et al., Eds.; MDText.com, Inc.: Bristol, MA, USA, 2000.

139. Tobinick, E.L.; Gross, H. Rapid cognitive improvement in Alzheimer's disease following perispinal etanercept administration. J. Neuroinflamm. 2008, 5, 2. [CrossRef]

140. Zhang, H.Y.; Yan, H.; Tang, X.C. Non-cholinergic effects of huperzine A: Beyond inhibition of acetylcholinesterase. Cell. Mol. Neurobiol. 2008, 28, 173-183. [CrossRef]

141. Marambaud, P.; Zhao, H.; Davies, P. Resveratrol promotes clearance of Alzheimer's disease amyloid-beta peptides. J. Biol. Chem. 2005, 280, 37377-37382. [CrossRef]

142. Kulkarni, S.K.; Dhir, A. Withania somnifera: An Indian ginseng. Prog. Neuropsychopharmacol. Biol. Psychiatry 2008, 32, 1093-1105. [CrossRef] [PubMed]

143. Luo, Y.; Smith, J.V.; Paramasivam, V.; Burdick, A.; Curry, K.J.; Buford, J.P.; Khan, I.; Netzer, W.J.; Xu, H.; Butko, P. Inhibition of amyloid-beta aggregation and caspase-3 activation by the Ginkgo biloba extract EGb761. Proc. Natl. Acad. Sci. USA 2002, 99, 12197-12202. [CrossRef] [PubMed]

144. Chen, F.; Eckman, E.A.; Eckman, C.B. Reductions in levels of the Alzheimer's amyloid beta peptide after oral administration of ginsenosides. FASEB J. 2006, 20, 1269-1271. [CrossRef] [PubMed]

145. Srinivasan, V.; Pandi-Perumal, S.R.; Cardinali, D.P.; Poeggeler, B.; Hardeland, R. Melatonin in Alzheimer's disease and other neurodegenerative disorders. Behav. Brain Funct. 2006, 2, 15. [CrossRef] [PubMed]

146. Janicki, S.C.; Schupf, N. Hormonal influences on cognition and risk for Alzheimer's disease. Curr. Neurol. Neurosci. Rep. 2010, 10, 359-366. [CrossRef] [PubMed]

147. Vrieling, A.; Buck, K.; Kaaks, R.; Chang-Claude, J. Adult weight gain in relation to breast cancer risk by estrogen and progesterone receptor status: A meta-analysis. Breast Cancer Res. Treat. 2010, 123, 641-649. [CrossRef] [PubMed]

148. Rabkin, J.G.; Quitkin, F.M.; McGrath, P.; Harrison, W.; Tricamo, E. Adverse reactions to monoamine oxidase inhibitors. Part II. Treatment correlates and clinical management. J. Clin. Psychopharmacol. 1985, 5, 2-9. [CrossRef] [PubMed]

149. Cai, Z. Monoamine oxidase inhibitors: Promising therapeutic agents for Alzheimer's disease (Review). Mol. Med. Rep. 2014, 9, 1533-1541. [CrossRef] [PubMed]

150. Pahan, K. Lipid-lowering drugs. Cell Mol. Life Sci. 2006, 63, 1165-1178. [CrossRef] [PubMed] 
151. Maji, D.; Shaikh, S.; Solanki, D.; Gaurav, K. Safety of statins. Indian J. Endocrinol. Metab. 2013, 17, $636-646$. [CrossRef] [PubMed]

152. Brooks, D.J. Dopamine agonists: Their role in the treatment of Parkinson's disease. J. Neurol. Neurosurg. Psychiatry 2000, 68, 685-689. [CrossRef]

153. Jankovic, J.; Aguilar, L.G. Current approaches to the treatment of Parkinson's disease. Neuropsychiatr. Dis. Treat. 2008, 4, 743-757. [CrossRef]

154. Bonifacio, M.J.; Palma, P.N.; Almeida, L.; Soares-da-Silva, P. Catechol-O-methyltransferase and its inhibitors in Parkinson's disease. CNS Drug Rev. 2007, 13, 352-379. [CrossRef]

155. Olanow, C.W. Tolcapone and hepatotoxic effects. Tasmar Advisory Panel. Arch. Neurol 2000, 57, $263-267$. [CrossRef]

156. Burkhard, P.; Dominici, P.; Borri-Voltattorni, C.; Jansonius, J.N.; Malashkevich, V.N. Structural insight into Parkinson's disease treatment from drug-inhibited DOPA decarboxylase. Nat. Struct. Biol. 2001, 8, 963-967. [CrossRef]

157. Youdim, M.B.; Bakhle, Y.S. Monoamine oxidase: Isoforms and inhibitors in Parkinson's disease and depressive illness. Br. J. Pharmacol. 2006, 147 (Suppl. 1), S287-S296. [CrossRef]

158. Brocks, D.R. Anticholinergic drugs used in Parkinson's disease: An overlooked class of drugs from a pharmacokinetic perspective. J. Pharm. Pharm. Sci. 1999, 2, 39-46.

159. Mizoguchi, K.; Yokoo, H.; Yoshida, M.; Tanaka, T.; Tanaka, M. Amantadine increases the extracellular dopamine levels in the striatum by re-uptake inhibition and by N-methyl-D-aspartate antagonism. Brain Res. 1994, 662, 255-258. [CrossRef]

160. Ratheesh, G.; Tian, L.; Venugopal, J.R.; Ezhilarasu, H.; Sadiq, A.; Fan, T.P.; Ramakrishna, S. Role of medicinal plants in neurodegenerative diseases. Biomanuf. Rev. 2017, 2, 2. [CrossRef]

161. Obrenovich, M.E.; Nair, N.G.; Beyaz, A.; Aliev, G.; Reddy, V.P. The role of polyphenolic antioxidants in health, disease, and aging. Rejuvenat. Res. 2010, 13, 631-643. [CrossRef]

162. Darvesh, A.S.; Carroll, R.T.; Bishayee, A.; Geldenhuys, W.J.; Van der Schyf, C.J. Oxidative stress and Alzheimer's disease: Dietary polyphenols as potential therapeutic agents. Expert Rev. Neurother. 2010, 10, 729-745. [CrossRef] [PubMed]

163. Smeriglio, A.; Barreca, D.; Bellocco, E.; Trombetta, D. Chemistry, Pharmacology and Health Benefits of Anthocyanins. Phytother. Res. 2016, 30, 1265-1286. [CrossRef] [PubMed]

164. Mazza, G.J. Anthocyanins and heart health. Annali-Istituto Superiore Di Sanita 2007, 43, 369-374.

165. Ali, T.; Rehman, S.U.; Shah, F.A.; Kim, M.O. Acute dose of melatonin via Nrf2 dependently prevents acute ethanol-induced neurotoxicity in the developing rodent brain. J. Neuroinflamm. 2018, 15, 119. [CrossRef] [PubMed]

166. Riaz, M.; Zia-Ul-Haq, M.; Saad, B. Anthocyanins and Human Health: Biomolecular and Therapeutic Aspects, Introduction to Anthocyanins; Springer: Basel, Switzerland, 2016.

167. Miguel, M.G. Anthocyanins: Antioxidant and/or anti-inflammatory activities. JAPS 2011, 1, 7-15.

168. Zhu, F.; Cai, Y.Z.; Yang, X.; Ke, J.; Corke, H. Anthocyanins, hydroxycinnamic acid derivatives, and antioxidant activity in roots of different chinese purple-fleshed sweetpotato genotypes. J. Agric. Food Chem. 2010, 58, 7588-7596. [CrossRef]

169. Zafra-Stone, S.; Yasmin, T.; Bagchi, M.; Chatterjee, A.; Vinson, J.A.; Bagchi, D. Berry anthocyanins as novel antioxidants in human health and disease prevention. Mol. Nutr. Food Res. 2007, 51, 675-683. [CrossRef]

170. Jeong, C.-H.; Jang, C.-W.; Kum, D.-C.; Yul Lee, K.; Yuan Lee, S.; Jin Hur, S.; Lee, S.-J. Protective effects of berry extracts on hydrogen peroxide-induced rat brain neuronal cell damage in vitro. J. Food Nutr. Res. 2014, 2, 277-280. [CrossRef]

171. Hwang, J.-W.; Kim, E.-K.; Lee, S.-J.; Kim, Y.-S.; Moon, S.-H.; Jeon, B.-T.; Sung, S.-H.; Kim, E.-T.; Park, P.-J. Antioxidant activity and protective effect of anthocyanin oligomers on $\mathrm{H}_{2} \mathrm{O}_{2}$-triggered $\mathrm{G} 2 / \mathrm{M}$ arrest in retinal cells. J. Agric. Food chem. 2012, 60, 4282-4288. [CrossRef]

172. Kelsey, N.; Hulick, W.; Winter, A.; Ross, E.; Linseman, D. Neuroprotective effects of anthocyanins on apoptosis induced by mitochondrial oxidative stress. Nutr. Neurosci. 2011, 14, 249-259. [CrossRef]

173. Lu, J.; Wu, D.M.; Zheng, Y.L.; Hu, B.; Zhang, Z.F. Purple sweet potato color alleviates d-galactose-induced brain aging in old mice by promoting survival of neurons via PI3K pathway and inhibiting cytochrome c-mediated apoptosis. Brain Pathol. 2010, 20, 598-612. [CrossRef] [PubMed] 
174. Toufektsian, M.-C.; De Lorgeril, M.; Nagy, N.; Salen, P.; Donati, M.B.; Giordano, L.; Mock, H.-P.; Peterek, S.; Matros, A.; Petroni, K. Chronic dietary intake of plant-derived anthocyanins protects the rat heart against ischemia-reperfusion injury. J. Nutr. 2008, 138, 747-752. [CrossRef] [PubMed]

175. Shih, P.H.; Yeh, C.T.; Yen, G.C. Anthocyanins induce the activation of phase II enzymes through the antioxidant response element pathway against oxidative stress-induced apoptosis. J. Agric. Food Chem. 2007, 55, 9427-9435. [CrossRef] [PubMed]

176. Hwang, Y.P.; Choi, J.H.; Yun, H.J.; Han, E.H.; Kim, H.G.; Kim, J.Y.; Park, B.H.; Khanal, T.; Choi, J.M.; Chung, Y.C.; et al. Anthocyanins from purple sweet potato attenuate dimethylnitrosamine-induced liver injury in rats by inducing Nrf2-mediated antioxidant enzymes and reducing COX-2 and iNOS expression. Food Chem. Toxicol. 2011, 49, 93-99. [CrossRef] [PubMed]

177. Steffen, Y.; Gruber, C.; Schewe, T.; Sies, H. Mono-O-methylated flavanols and other flavonoids as inhibitors of endothelial NADPH oxidase. Arch. Biochem. Biophys. 2008, 469, 209-219. [CrossRef] [PubMed]

178. Heaney, R.P. Factors influencing the measurement of bioavailability, taking calcium as a model. J. Nutr. 2001, 131, 1344s-1348s. [CrossRef] [PubMed]

179. Kay, C.D.; Pereira-Caro, G.; Ludwig, I.A.; Clifford, M.N.; Crozier, A. Anthocyanins and Flavanones Are More Bioavailable than Previously Perceived: A Review of Recent Evidence. Ann. Rev. Food Sci. Technol. 2017, 8, 155-180. [CrossRef] [PubMed]

180. He, B.; Ge, J.; Yue, P.; Yue, X.; Fu, R.; Liang, J.; Gao, X. Loading of anthocyanins on chitosan nanoparticles influences anthocyanin degradation in gastrointestinal fluids and stability in a beverage. Food Chem. 2017, 221, 1671-1677. [CrossRef] [PubMed]

181. Amin, F.U.; Shah, S.A.; Badshah, H.; Khan, M.; Kim, M.O. Anthocyanins encapsulated by PLGA@PEG nanoparticles potentially improved its free radical scavenging capabilities via p38/JNK pathway against Abeta1-42-induced oxidative stress. J. Nanobiotechnol. 2017, 15, 12. [CrossRef]

182. Kim, M.J.; Rehman, S.U.; Amin, F.U.; Kim, M.O. Enhanced neuroprotection of anthocyanin-loaded PEG-gold nanoparticles against Abeta1-42-induced neuroinflammation and neurodegeneration via the NF-KB /JNK/GSK3beta signaling pathway. Nanomedicine 2017, 13, 2533-2544. [CrossRef]

183. He, J.; Giusti, M.M. Anthocyanins: Natural colorants with health-promoting properties. Ann. Rev. Food Sci. Technol. 2010, 1, 163-187. [CrossRef] [PubMed]

184. Celli, G.B.; Tan, C.; Selig, M.J. Anthocyanidins and Anthocyanins. Food Sci. 2019, 218-223. [CrossRef]

185. Bartikova, H.; Skalova, L.; Drsata, J.; Bousova, I. Interaction of anthocyanins with drug-metabolizing and antioxidant enzymes. Curr. Med. Chem. 2013, 20, 4665-4679. [CrossRef] [PubMed]

186. Shukitt-Hale, B.; Kalt, W.; Carey, A.N.; Vinqvist-Tymchuk, M.; McDonald, J.; Joseph, J.A. Plum juice, but not dried plum powder, is effective in mitigating cognitive deficits in aged rats. Nutrition 2009, 25, 567-573. [CrossRef] [PubMed]

187. Tsuda, T. Dietary anthocyanin-rich plants: Biochemical basis and recent progress in health benefits studies. Mol. Nutr. Food Res. 2012, 56, 159-170. [CrossRef] [PubMed]

188. Khan, M.; Shah, S.A.; Kim, M.O. 17beta-Estradiol via SIRT1/Acetyl-p53/NF-kB Signaling Pathway Rescued Postnatal Rat Brain Against Acute Ethanol Intoxication. Mol. Neurobiol. 2018, 55, 3067-3078. [CrossRef]

189. Rahman, M.M.; Ichiyanagi, T.; Komiyama, T.; Sato, S.; Konishi, T. Effects of anthocyanins on psychological stress-induced oxidative stress and neurotransmitter status. J. Agric. Food Chem. 2008, 56, 7545-7550. [CrossRef]

190. Ye, J.; Meng, X.; Yan, C.; Wang, C. Effect of purple sweet potato anthocyanins on beta-amyloid-mediated PC-12 cells death by inhibition of oxidative stress. Neurochem. Res. 2010, 35, 357-365. [CrossRef]

191. Kang, T.H.; Hur, J.Y.; Kim, H.B.; Ryu, J.H.; Kim, S.Y. Neuroprotective effects of the cyanidin-3-O-beta-d-glucopyranoside isolated from mulberry fruit against cerebral ischemia. Neurosci. Lett. 2006, 391, 122-126. [CrossRef]

192. Krikorian, R.; Nash, T.A.; Shidler, M.D.; Shukitt-Hale, B.; Joseph, J.A. Concord grape juice supplementation improves memory function in older adults with mild cognitive impairment. Br. J. Nutr. 2010, 103, 730-734. [CrossRef]

193. Joseph, J.A.; Denisova, N.A.; Arendash, G.; Gordon, M.; Diamond, D.; Shukitt-Hale, B.; Morgan, D. Blueberry supplementation enhances signaling and prevents behavioral deficits in an Alzheimer disease model. Nutr. Neurosci. 2003, 6, 153-162. [CrossRef] [PubMed] 
194. Ullah, I.; Park, H.Y.; Kim, M.O. Anthocyanins protect against kainic acid-induced excitotoxicity and apoptosis via ROS-activated AMPK pathway in hippocampal neurons. CNS Neurosci. Ther. 2014, 20, 327-338. [CrossRef] [PubMed]

195. Shah, S.A.; Yoon, G.H.; Kim, M.O. Protection of the developing brain with anthocyanins against ethanol-induced oxidative stress and neurodegeneration. Mol. Neurobiol. 2015, 51, 1278-1291. [CrossRef] [PubMed]

196. Rehman, S.U.; Shah, S.A.; Ali, T.; Chung, J.I.; Kim, M.O. Anthocyanins Reversed D-Galactose-Induced Oxidative Stress and Neuroinflammation Mediated Cognitive Impairment in Adult Rats. Mol. Neurobiol. 2017, 54, 255-271. [CrossRef]

197. Khan, M.S.; Ali, T.; Kim, M.W.; Jo, M.H.; Jo, M.G.; Badshah, H.; Kim, M.O. Anthocyanins protect against LPS-induced oxidative stress-mediated neuroinflammation and neurodegeneration in the adult mouse cortex. Neurochem. Int. 2016, 100, 1-10. [CrossRef] [PubMed]

198. Ali, T.; Kim, T.; Rehman, S.U.; Khan, M.S.; Amin, F.U.; Khan, M.; Ikram, M.; Kim, M.O. Natural Dietary Supplementation of Anthocyanins via PI3K/Akt/Nrf2/HO-1 Pathways Mitigate Oxidative Stress, Neurodegeneration, and Memory Impairment in a Mouse Model of Alzheimer's Disease. Mol. Neurobiol. 2017. [CrossRef] [PubMed]

199. Khan, M.S.; Ali, T.; Kim, M.W.; Jo, M.H.; Chung, J.I.; Kim, M.O. Anthocyanins Improve Hippocampus-Dependent Memory Function and Prevent Neurodegeneration via JNK/Akt/GSK3beta Signaling in LPS-Treated Adult Mice. Mol. Neurobiol. 2019, 56, 671-687. [CrossRef] [PubMed]

200. Chao, J.; Leung, Y.; Wang, M.; Chang, R.C. Nutraceuticals and their preventive or potential therapeutic value in Parkinson's disease. Nutr. Rev. 2012, 70, 373-386. [CrossRef]

201. Albarracin, S.L.; Stab, B.; Casas, Z.; Sutachan, J.J.; Samudio, I.; Gonzalez, J.; Gonzalo, L.; Capani, F.; Morales, L.; Barreto, G.E. Effects of natural antioxidants in neurodegenerative disease. Nutr. Neurosci. 2012, 15, 1-9. [CrossRef] [PubMed]

202. Guo, S.; Yan, J.; Yang, T.; Yang, X.; Bezard, E.; Zhao, B. Protective effects of green tea polyphenols in the 6-OHDA rat model of Parkinson's disease through inhibition of ROS-NO pathway. Biol. Psychiatry 2007, 62, 1353-1362. [CrossRef] [PubMed]

203. Zbarsky, V.; Datla, K.P.; Parkar, S.; Rai, D.K.; Aruoma, O.I.; Dexter, D.T. Neuroprotective properties of the natural phenolic antioxidants curcumin and naringenin but not quercetin and fisetin in a 6-OHDA model of Parkinson's disease. Free Radic. Res. 2005, 39, 1119-1125. [CrossRef] [PubMed]

204. Chao, J.; Yu, M.S.; Ho, Y.S.; Wang, M.; Chang, R.C. Dietary oxyresveratrol prevents parkinsonian mimetic 6-hydroxydopamine neurotoxicity. Free Radic. Biol. Med. 2008, 45, 1019-1026. [CrossRef] [PubMed]

205. Khan, M.M.; Ahmad, A.; Ishrat, T.; Khan, M.B.; Hoda, M.N.; Khuwaja, G.; Raza, S.S.; Khan, A.; Javed, H.; Vaibhav, K.; et al. Resveratrol attenuates 6-hydroxydopamine-induced oxidative damage and dopamine depletion in rat model of Parkinson's disease. Brain Res. 2010, 1328, 139-151. [CrossRef] [PubMed]

206. Song, J.X.; Sze, S.C.; Ng, T.B.; Lee, C.K.; Leung, G.P.; Shaw, P.C.; Tong, Y.; Zhang, Y.B. Anti-Parkinsonian drug discovery from herbal medicines: What have we got from neurotoxic models? J. Ethnopharmacol. 2012, 139, 698-711. [CrossRef] [PubMed]

207. Gao, X.; Cassidy, A.; Schwarzschild, M.A.; Rimm, E.B.; Ascherio, A. Habitual intake of dietary flavonoids and risk of Parkinson disease. Neurology 2012, 78, 1138-1145. [CrossRef]

208. Strathearn, K.E.; Yousef, G.G.; Grace, M.H.; Roy, S.L.; Tambe, M.A.; Ferruzzi, M.G.; Wu, Q.L.; Simon, J.E.; Lila, M.A.; Rochet, J.C. Neuroprotective effects of anthocyanin- and proanthocyanidin-rich extracts in cellular models of Parkinsons disease. Brain Res. 2014, 1555, 60-77. [CrossRef]

209. Ali, T.; Kim, M.J.; Rehman, S.U.; Ahmad, A.; Kim, M.O. Anthocyanin-Loaded PEG-Gold Nanoparticles Enhanced the Neuroprotection of Anthocyanins in an Abeta1-42 Mouse Model of Alzheimer's Disease. Mol. Neurobiol. 2017, 54, 6490-6506. [CrossRef]

210. Opinion, S. Scientific Opinion on the re-evaluation of anthocyanins (E 163) as a food additive. EFSA J. 2013, 11, 3145. [CrossRef]

211. Pojer, E.; Mattivi, F.; Johnson, D.; Stockley, C.S. The Case for Anthocyanin Consumption to Promote Human Health: A Review. Compr. Rev. Food Sci. Food Saf. 2013, 12, 483-508. [CrossRef]

212. Niki, E.; Yamamoto, Y.; Takahashi, M.; Yamamoto, K.; Yamamoto, Y.; Komuro, E.; Miki, M.; Yasuda, H.; Mino, M. Free radical-mediated damage of blood and its inhibition by antioxidants. J. Nutr. Sci. Vitaminol. (Tokyo) 1988, 34, 507-512. [CrossRef] 
213. Rossetto, M.; Vanzani, P.; Mattivi, F.; Lunelli, M.; Scarpa, M.; Rigo, A. Synergistic antioxidant effect of catechin and malvidin 3-glucoside on free radical-initiated peroxidation of linoleic acid in micelles. Arch. Biochem. Biophys. 2002, 408, 239-245. [CrossRef]

214. Markesbery, W.R. Oxidative stress hypothesis in Alzheimer's disease. Free Radic. Biol. Med. 1997, $23,134-147$. [CrossRef]

215. Kanowski, S.; Hoerr, R. Ginkgo biloba extract EGb 761 in dementia: Intent-to-treat analyses of a 24-week, multi-center, double-blind, placebo-controlled, randomized trial. Pharmacopsychiatry 2003, 36, 297-303. [CrossRef] [PubMed]

216. Fahn, S. An open trial of high-dosage antioxidants in early Parkinson's disease. Am. J. Clin. Nutr. 1991, 53, 380s-382s. [CrossRef] [PubMed]

(C) 2019 by the authors. Licensee MDPI, Basel, Switzerland. This article is an open access article distributed under the terms and conditions of the Creative Commons Attribution (CC BY) license (http://creativecommons.org/licenses/by/4.0/). 